\title{
Partition of electronic excitation energies: the IQA/EOM-CCSD method
}

\author{
Alberto Fernández-Alarcón ${ }^{\mathrm{a}, \mathrm{b}}$, José Luis Casals-Sainz ${ }^{\mathrm{b}}$, José Manuel Guevara-Velab , Aurora \\ Costales $^{\mathrm{b}}$, Evelio Francisco ${ }^{\mathrm{b}}$, Ángel Martín Pendás ${ }^{\mathrm{b}}$, Tomás Rocha Rinza ${ }^{\mathrm{a}, *}$ \\ ${ }^{a}$ Universidad Nacional Autónoma de México, Instituto de Química, Ciudad Universitaria, Ciudad de \\ México, 04510 México \\ ${ }^{b}$ Departament of Analytical and Physical Chemistry, University of Oviedo, E-33006, Oviedo, Spain.
}

\begin{abstract}
Different developments in chemistry and emerging technologies have generated a renewed interest in the properties of molecular excited states. We present herein the partition of black-box, size-consistent Equation-Of-Motion Coupled Cluster Singles and Doubles (EOMCCSD) excitation energies within the framework of the Interacting Quantum Atoms (IQA) formalism. We denote this method as IQA/EOM-CCSD. We illustrate this approach by considering small molecules used often in the study of excited states. This investigation shows how the combination of IQA and EOM-CCSD may provide valuable insights on the molecular changes induced by electron excitation via the real space distribution of the energy of an absorbed photon in a molecular system. Our results reveal (i) the most energetically deformed atomic basins and (ii) the most affected covalent and non-covalent interactions within a molecule due to a given photoexcitation. In other words, this kind of analysis provides insights about the spatial energetic redistribution accompanying an electronic excitation, with interesting foreseeable applications in the rational design of photoexcitations with tailored chemical effects. Altogether, we expect that the IQA/EOM-CCSD excitation energy partition will prove useful in the understanding of systems and processes of interest in photophysics and photochemistry.
\end{abstract}

Keywords:

Partition of the excitation energy, Interacting Quantum Atoms, EOM-CCSD.

${ }^{*}$ To whom correspondence should be addressed: tomasrocharinza@gmail.com 


\section{Introduction}

The study of molecules and molecular clusters in excited states has opened exciting possibilities in organic and inorganic chemistry. For example, photoexcitation might induce (i) a substantial increase in acidity $^{[1,2]}$ (ii) ultrafast intramolecular proton transfers ${ }^{[3]}$, (iii) isomerisations $^{[4,5]}$, (iv) polymerisations using incident intensities reduced in two orders of magnitude relative to those of usual initiators ${ }^{[6]}$, and (v) changes in chemical structure which can be used in the design of molecular switches ${ }^{[7]}$ among many other effects. Besides these advances, our understanding of physical and chemical processes of molecules in excited states will play a critical role in the development of emergent technologies, like the production of photovoltaic cells $^{[8,9]}$, the use of artificial photosynthesis to generate biofuel ${ }^{[10]}$ and biological detection and imaging ${ }^{[11]}$.

The above-mentioned circumstances warrant continuous efforts to gain further insights about the properties and behaviour of electronic systems in excited states. Hereof, Quantum Chemical Topology (QCT) ${ }^{[12]}$ has provided different tools for the understanding of many molecules and non-covalent complexes. ${ }^{[13-18]}$ Among the QCT approaches, the Interacting Quantum Atoms (IQA) yields a physically-sound partition of the electronic energy based solely on the vector state of the system of interest ${ }^{[19,20]}$. Despite the many successful applications of IQA in Ground State (GS), ${ }^{[21-27]}$ there are only a few studies wherein this methodology is exploited to investigate the effects of photoexcitation on molecules ${ }^{[28,29]}$. These previous applications of the IQA partitions were based on $\mathrm{CASSCF}^{[29]}$ and later on MR-CISD ${ }^{[28]}$ wavefunctions. Although these methods are very important in computational chemistry, the former does not include a substantial contribution of dynamical correlation while the latter yields electronic energies that are not size-consistent and its non-black-box use requires many timeconsuming exploratory calculations for its successful application ${ }^{[30]}$. It is therefore desirable to count on energy partitions of correlated, black-box, size-consistent, quantum-chemical approximations applicable to the study of electronic excited states. The availability of this type of methods of wavefunction analyses would provide a chance to dissect not only excitation energies themselves, $\Delta E=h \nu$, but also the influence on $\Delta E$ due to different circumstances such as non-covalent interactions, or the occurrence of certain functional groups in the system 
of interest.

Given this background, we introduce herein the partition of excitation energies computed with the Equation-Of-Motion Coupled Cluster Singles and Doubles (EOM-CCSD) approximation in one- and two- particle terms according to the IQA formalism, i.e., the IQA/EOMCCSD method. This division of $\Delta E$ will give information concerning which are the most importantly affected atoms and atomic interactions (viz. covalent and non-covalent chemical bonds) within a molecule or molecular adduct because of electron excitation and therefore to assess whether photoabsorption has an intended effect on a given electronic system.

This article is divided as follows. We give first a brief account of the IQA method of wavefunction analysis and the resulting partition of the excitation energy. Then, we provide a short revision of Coupled Cluster (CC) and EOM-CC theories, putting special emphasis on the computation of the density functions necessary to perform the real space partition of excitation energies proposed in this research, to later present the computational details of our calculations. We illustrate subsequently the IQA/EOM-CCSD method with small molecules

utilized for the study of excited states: homo- and heteronuclear diatomic molecules and noble gas excimers, species with basic lone pairs as well as saturated and unsaturated hydrocarbons. Overall, we expect that the IQA/EOM-CCSD approach will prove a valuable tool for the examination of the changes induced by photoexcitation of molecules and non-covalent clusters in photosciences.

\section{Theory}

\subsection{IQA energy splitting scheme}

The IQA energy partition scheme is a relevant method of wave function analysis in the QCT field, which is originally based on the Quantum Theory of Atoms in Molecules (QTAIM) division of real space. The importance of IQA resides not only on its recovery of the total electronic energy depending solely on the vector state of a given system but also on its detailed description of the interactions between atoms or functional groups inside molecules and molecular clusters. Briefly, the IQA splitting considers the electronic Born-Oppenheimer energy, 


$$
\begin{aligned}
E & =\int \widehat{h} \rho_{1}\left(\mathbf{r}_{1}, \mathbf{r}_{1}^{\prime}\right) \mathrm{d} \mathbf{r}_{1}+\frac{1}{2} \iint \frac{\rho_{2}\left(\mathbf{r}_{1}, \mathbf{r}_{2}\right)}{r_{12}} \mathrm{~d} \mathbf{r}_{1} \mathrm{~d} \mathbf{r}_{2}+E_{\text {nuc }} \\
& =\sum_{\mathrm{A}} \int_{\mathrm{A}} \widehat{h} \rho_{1}\left(\mathbf{r}_{1}, \mathbf{r}_{1}^{\prime}\right) \mathrm{d} \mathbf{r}_{1}+\frac{1}{2} \sum_{\mathrm{AB}} \int_{\mathrm{A}} \int_{\mathrm{B}} \frac{\rho_{2}\left(\mathbf{r}_{1}, \mathbf{r}_{2}\right)}{r_{12}} \mathrm{~d} \mathbf{r}_{1} \mathrm{~d} \mathbf{r}_{2}+\sum_{\mathrm{A}>\mathrm{B}} \frac{Z_{\mathrm{A}} Z_{\mathrm{B}}}{R_{\mathrm{AB}}},
\end{aligned}
$$

in which $\widehat{h}$ represents the monoelectronic operators of the electronic Hamiltonian, $Z_{\mathrm{X}}$ is the atomic number of nucleus $\mathrm{X}, E_{\text {nuc }}$ the nuclear repulsion of the system and $\rho_{1}\left(\mathbf{r}_{1}, \mathbf{r}_{1}^{\prime}\right)$ and $\rho_{2}\left(\mathbf{r}_{1}, \mathbf{r}_{2}\right)$ denote the reduced first order density matrix and the pair density of the system under consideration respectively. The summations in the second equality of expression (1) are done over the regions $\mathrm{A}, \mathrm{B}, \ldots$ of an exhaustive partition of the three dimensional space such as the QTAIM basins. By collecting one- and two-basin terms in expression (1) we get,

$$
\begin{aligned}
E & =\sum_{\mathrm{A}} E_{\mathrm{net}}^{\mathrm{A}}+\sum_{\mathrm{A}>\mathrm{B}} E_{\mathrm{int}}^{\mathrm{AB}} \\
& =\sum_{\mathrm{A}}\left(T^{\mathrm{A}}+E_{\mathrm{ne}}^{\mathrm{AA}}+E_{\mathrm{ee}}^{\mathrm{AA}}\right)+\sum_{\mathrm{A}>\mathrm{B}}\left(E_{\mathrm{nn}}^{\mathrm{AB}}+E_{\mathrm{ne}}^{\mathrm{AB}}+E_{\mathrm{ne}}^{\mathrm{BA}}+E_{\mathrm{ee}}^{\mathrm{AB}}\right),
\end{aligned}
$$

in which $E_{\text {net }}^{\mathrm{A}}$ and $E_{\text {int }}^{\mathrm{AB}}$ are the net energies of atom $\mathrm{A}$ and the interaction energy of pair $\mathrm{AB}$ respectively. Additionally, $T^{\mathrm{A}}$ denotes the kinetic energy of atom $\mathrm{A}$, while $E_{\sigma \tau}^{\mathrm{AB}}$ represents the interaction energy between $\sigma$ in atom $\mathrm{A}$ and $\tau$ in atom $\mathrm{B}$, wherein $\sigma$ and $\tau$ represent either a nucleus or electrons. These components of the IQA energy partition are given by

$$
\begin{aligned}
T^{\mathrm{A}} & =-\frac{1}{2} \int_{\mathrm{A}} \nabla^{2} \rho_{1}\left(\mathbf{r}_{1}, \mathbf{r}_{1}^{\prime}\right) \mathrm{d} \mathbf{r}_{1}, \\
E_{\mathrm{ne}}^{\mathrm{AB}} & =-Z_{\mathrm{A}} \int_{\mathrm{B}} \frac{\rho_{1}\left(\mathbf{r}_{1}\right)}{r_{1 \mathrm{~A}}} \mathrm{~d} \mathbf{r}_{1}, \\
E_{\mathrm{ee}}^{\mathrm{AB}} & =\frac{2-\delta_{\mathrm{AB}}}{2} \int_{\mathrm{A}} \int_{\mathrm{B}} \frac{\rho_{2}\left(\mathbf{r}_{1}, \mathbf{r}_{1}\right)}{r_{12}} \mathrm{~d} \mathbf{r}_{1} \mathrm{~d} \mathbf{r}_{2} .
\end{aligned}
$$

The interaction energy term can be further split as the sum of classical and exchange- 
correlation contributions,

$$
\begin{aligned}
E_{\mathrm{int}}^{\mathrm{AB}} & =E_{\text {class }}^{\mathrm{AB}}+E_{\mathrm{xc}}^{\mathrm{AB}} \\
& =E_{\mathrm{nn}}^{\mathrm{AB}}+E_{\mathrm{ne}}^{\mathrm{AB}}+E_{\mathrm{ne}}^{\mathrm{BA}}+E_{\mathrm{j}}^{\mathrm{AB}}+E_{\mathrm{xc}}^{\mathrm{AB}},
\end{aligned}
$$

where,

$$
\begin{aligned}
& E_{\mathrm{j}}^{\mathrm{AB}}=\int_{\mathrm{A}} \int_{\mathrm{B}} \frac{\rho_{1}\left(\mathbf{r}_{1}\right) \rho_{1}\left(\mathbf{r}_{2}\right)}{r_{12}} \mathrm{~d} \mathbf{r}_{1} \mathrm{~d} \mathbf{r}_{2}, \\
& E_{\mathrm{xc}}^{\mathrm{AB}}=\int_{\mathrm{A}} \int_{\mathrm{B}} \frac{\rho_{2}\left(\mathbf{r}_{1}, \mathbf{r}_{2}\right)-\rho_{1}\left(\mathbf{r}_{1}\right) \rho_{1}\left(\mathbf{r}_{2}\right)}{r_{12}} \mathrm{~d} \mathbf{r}_{1} \mathrm{~d} \mathbf{r}_{2} .
\end{aligned}
$$

We introduce at this point a QTAIM-derived concept, the delocalisation index between two atoms $\mathrm{A}$ and $\mathrm{B}$ and hereby denoted as $\mathrm{DI}(\mathrm{A}, \mathrm{B})$, which is in close relationship with $E_{\mathrm{xc}}^{\mathrm{AB}}$. Delocalisation indexes are indicators of the number of shared pairs of electrons between two atoms. ${ }^{[31,32]}$ These indices are computed as the absolute value of the integral of the Fermi and Coulomb hole,

$$
\mathrm{DI}(\mathrm{A}, \mathrm{B})=\int_{\mathrm{A}} \int_{\mathrm{B}}\left(\rho_{2}\left(\mathbf{r}_{1}, \mathbf{r}_{2}\right)-\rho_{1}\left(\mathbf{r}_{1}\right) \rho_{1}\left(\mathbf{r}_{2}\right)\right) \mathrm{d} \mathbf{r}_{1} \mathrm{~d} \mathbf{r}_{2}
$$

Notice the similarity of DI(A, B) in formula (9) and the exchange-correlation energy between these two atoms, $E_{\mathrm{xc}}^{\mathrm{AB}}$ in expression (8). The connection between these quantities has been examined before. ${ }^{[33]}$ 
Finally, as we can see in expressions (3)-(8), we need first and second order density functions to perform the IQA analysis. These scalar fields are given by,

$$
\begin{aligned}
\rho_{1}\left(\mathbf{r}_{1}, \mathbf{r}_{1}^{\prime}\right) & =N \int \Psi^{*}\left(\mathbf{x}_{1}^{\prime}, \mathbf{x}_{2}, \ldots, \mathbf{x}_{N}\right) \Psi\left(\mathbf{x}_{1}, \mathbf{x}_{2}, \ldots, \mathbf{x}_{N}\right) \mathrm{d} s_{1} \mathrm{~d} s_{1}^{\prime} \mathrm{d} \mathbf{x}_{2} \ldots \mathrm{d} \mathbf{x}_{N} \\
& =\sum_{p q} D_{p q} \phi_{p}^{*}\left(\mathbf{r}_{1}^{\prime}\right) \phi_{q}\left(\mathbf{r}_{1}\right), \text { and }, \\
\rho_{2}\left(\mathbf{r}_{1}, \mathbf{r}_{2}\right) & =N(N-1) \int \Psi^{*}\left(\mathbf{x}_{1}, \mathbf{x}_{2}, \ldots, \mathbf{x}_{N}\right) \Psi\left(\mathbf{x}_{1}, \mathbf{x}_{2}, \ldots, \mathbf{x}_{2}\right) \mathrm{d} s_{1} \mathrm{~d} s_{2} \mathrm{~d} \mathbf{x}_{3} \ldots \mathrm{d} \mathbf{x}_{N} \\
& =\sum_{p q r s} d_{p q r s} \phi_{p}^{*}\left(\mathbf{r}_{1}\right) \phi_{q}\left(\mathbf{r}_{1}\right) \phi_{r}^{*}\left(\mathbf{r}_{2}\right) \phi_{s}\left(\mathbf{r}_{2}\right),
\end{aligned}
$$

wherein $B=\left\{\phi_{p}\right\}$ is the basis set which underlies the Fock space of the system of interest and it is usually chosen as Hartree-Fock canonical orbitals on one hand, while on the other $\mathbf{D}$ and $\mathbf{d}$ are the one- and two-electron matrices in the basis set $B$. Once determined the $\rho_{1}\left(\mathbf{r}_{1}, \mathbf{r}^{\prime}\right)$ and $\rho_{2}\left(\mathbf{r}_{1}, \mathbf{r}_{2}\right)$ scalar fields, we can compute the different contributions to $E_{\text {net }}^{\mathrm{A}}$ (formulae (3)-(5) as well as the components of $E_{\mathrm{int}}^{\mathrm{AB}}$ with aid of equations (7)-(8). In other words, the coupling between the IQA partition and any electronic structure method approximation can be done by means of the computation of the $\mathbf{D}$ and $\mathbf{d}$ matrices in a given basis set. ${ }^{1}$

Next, we consider CC and EOM-CC theories to define the $D_{p q}$ and $d_{p q r s}$ matrix elements in the latter approximation, to subsequently compute the density functions necessary to perform the IQA/EOM-CCSD partition of excitation energies.

\footnotetext{
${ }^{1}$ The one- and two-electron matrices $\mathbf{D}$ and $\mathbf{d}$ comprise discrete representations of the first and second order reduced density matrices in the orthonormal basis set $\left\{\phi_{p}\right\}{ }^{\left[{ }^{[34]}\right.}$ As stated above, this orthonormal basis is normally (and conveniently) taken as the Hartree-Fock canonical orbitals. The one $\left(D_{p q}\right)$ and two electron matrix elements $\left(d_{p q r s}\right)$ which satisfy respectively equations (10) and (11) are given by

$$
\begin{aligned}
D_{p q} & =\iint \phi_{p}\left(\mathbf{r}_{1}^{\prime}\right) \rho_{1}\left(\mathbf{r}_{1}, \mathbf{r}_{1}^{\prime}\right) \phi_{q}^{*}\left(\mathbf{r}_{1}\right) \mathrm{d} \mathbf{r}_{1}^{\prime} \mathrm{d} \mathbf{r}_{1}, \\
d_{p q r s} & =\iint \phi_{p}\left(\mathbf{r}_{1}\right) \phi_{q}^{*}\left(\mathbf{r}_{1}\right) \rho_{2}\left(\mathbf{r}_{1}, \mathbf{r}_{2}\right) \phi_{r}\left(\mathbf{r}_{2}\right) \phi_{s}^{*}\left(\mathbf{r}_{2}\right) \mathrm{d} \mathbf{r}_{1} \mathrm{~d} \mathbf{r}_{2} .
\end{aligned}
$$




\subsection{The $C C$ method}

Coupled cluster (CC) theory is based on the idea that electronic correlation results in a virtual excitation of a group of electrons (a cluster of one, two, .., $N$ particles) from occupied $(i, j, k, .$.$) to virtual (a, b, c, \ldots)$ orbitals. The $\mathrm{CC}$ wavefunction can be written as,

$$
|\mathrm{CC}\rangle=e^{\widehat{T}}|0\rangle
$$

where $|0\rangle$ is a reference state which is usually chosen as the Hartree-Fock determinant, and the CC operator $\widehat{T}$ is

$$
\widehat{T}=\sum_{m} \widehat{T}_{m}
$$

with

$$
\begin{aligned}
\widehat{T}_{m} & =\sum_{\substack{a_{1}<a_{2}<\ldots<a_{m} \\
i_{1}<i_{2}<\ldots<i_{m}}} t_{i_{1}, a_{2}, \ldots, i_{m}}^{a_{1}, a_{2}, \ldots, a_{m}} \widehat{\tau}_{1} \widehat{\tau}_{2} \ldots \widehat{\tau}_{m} \\
& =\sum_{\substack{a_{1}<a_{2}<\ldots<a_{m}, i_{2}, \ldots, i_{m} \\
i_{1}<i_{2}<\ldots<i_{m}}} t_{1}^{a_{1}, a_{2}, \ldots, a_{m}} \widehat{a}_{1}^{\dagger} \widehat{i}_{1} \widehat{a}_{2}^{\dagger} \widehat{i}_{2} \ldots \widehat{a}_{m}^{\dagger} \widehat{i}_{m},
\end{aligned}
$$

in which $\widehat{p}$ and $\widehat{q}^{\dagger}$ represent general annihilation and creation operators respectively. As customarily used, the dagger distinguish creation from annihilation operators. In this way, the operator $\widehat{a}_{k}^{\dagger}$ creates a particle in the virtual orbital $a_{k}$ and $\widehat{i}_{k}$ annihilates a particle in the $i_{k}$ occupied orbital. The symbol $t_{i_{1}, i_{2}, \ldots, i_{m}}^{a_{1}, a_{2}, \ldots, a_{m}}$ is the cluster amplitude related to the $m$-tuple excitation $\left(i_{1}, \ldots, i_{m}\right) \rightarrow\left(a_{1}, \ldots, a_{m}\right)$. The truncation $\widehat{T}=\widehat{T}_{1}+\widehat{T}_{2}$ defines the Coupled Cluster Singles and Doubles (CCSD) approximation. We use herein the normal-ordered electronic Hamiltonian $\widehat{H}_{N}$ defined in the language of second quantisation as

$$
\begin{aligned}
\widehat{H}_{N} & =\widehat{H}-\langle 0|\widehat{H}| 0\rangle \\
& =\sum_{p q} f_{p q} \widehat{p}^{\dagger} \widehat{q}+\frac{1}{4} \sum_{p q r s} g_{p q r s} \widehat{p}^{\dagger} \widehat{q}^{\dagger} \widehat{s} \widehat{r}
\end{aligned}
$$

where $f_{p q}$ are the Fock matrix elements and $g_{p q r s}=\langle p r \| q s\rangle$ denote the antisymmetrised two electron integrals in the basis $B$. To calculate the ground state $\mathrm{CC}$ correlation energy, 
$E_{\text {corr }}^{\mathrm{CC}}$, and the cluster amplitudes, it is necessary to solve the projected CC equations. These equations are the result of applying the Hamiltonian $\widehat{H}_{N}$ to the CC wave function (14), then multiplying from the left by $e^{-\widehat{T}}$ to finally project onto the vacuum state $|0\rangle$ and the excited determinants space $\left|{ }_{i_{1} i_{2} \ldots}^{a_{1} a_{2} \ldots}\right\rangle=\widehat{a}_{1}^{\dagger} \widehat{i}_{1} \widehat{a}_{2}^{\dagger} \widehat{i}_{2} \ldots|0\rangle$ to obtain,

$$
\begin{aligned}
& \left\langle 0\left|e^{-\widehat{T}} \widehat{H}_{N} e^{\widehat{T}}\right| 0\right\rangle=E_{\text {corr }}^{\mathrm{CC}} \text {, and, }
\end{aligned}
$$

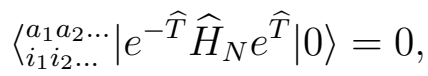

in which when $\widehat{T}=\widehat{T}_{1}+\widehat{T}_{2}$, we refer $E_{\text {corr }}^{\mathrm{CC}}$ as $E_{\text {corr }}^{\mathrm{CCSD}}$.

\subsection{EOM-CC theory}

The equation of motion coupled cluster method was developed as an extension of CC theory to describe electronic systems in excited states. In particular, the EOM-CCSD wave functions are described by linear combinations of excitation operators applied to a CCSD reference state. We have thus for the $k$-th excited state,

$$
\begin{aligned}
|k\rangle & =\widehat{\mathcal{R}}_{k} e^{\widehat{T}}|0\rangle \\
& =e^{\widehat{T}} \widehat{\mathcal{R}}_{k}|0\rangle .
\end{aligned}
$$

As mentioned above, the operator $\widehat{\mathcal{R}}_{k}$ equals a linear combination of excitation operators,

$$
\widehat{\mathcal{R}}_{k}=r_{0}+\sum_{i_{1}, a_{1}} r_{i_{1}}^{a_{1}} \widehat{a}_{1} \widehat{i}_{1}+\sum_{\substack{a_{1}<a_{2} \\ i_{1}<i_{2}}} r_{i_{1} i_{2}}^{a_{1} a_{2}} \widehat{a}_{1}^{\dagger} \hat{i_{1}} \widehat{a}_{2}^{\dagger} \widehat{i}_{2}+\ldots
$$

After introducing equation (20) in the Schrödinger equation with the normal-ordered Hamiltonian, and multiplying by $e^{-\widehat{T}}$ from the left, we obtain the equations for the EOM-CCSD right eigenvectors,

$$
e^{-\widehat{T}} \widehat{H}_{\tilde{N}} e^{\widehat{T}} \widehat{\mathcal{R}}_{k}|0\rangle=\Delta E_{k}^{\mathrm{EOM}-\mathrm{CCSD}} \widehat{\mathcal{R}}_{k}|0\rangle,
$$


in which $\widehat{H}_{\tilde{N}}=\widehat{H}_{N}-E_{\text {corr }}^{\mathrm{CCSD}}$ and $\Delta E_{k}^{\mathrm{EOM}-\mathrm{CCSD}}$ is the EOM-CCSD excitation energy for the $k$-th excited state and both operators $\widehat{T}$ and $\widehat{\mathcal{R}}_{k}$ include only single and double excitations. Equation (22) shows that EOM-CCSD photoexcitation energies are determined by diagonalising the matrix representation of the operator $e^{-\widehat{T}} \widehat{H}_{\tilde{N}} e^{\widehat{T}} \cdot{ }^{[35]}$ The non-Hermitian nature of the operator $e^{-\widehat{T}} \widehat{H}_{\tilde{N}} e^{\widehat{T}}$ ( $\widehat{T}$ is not an antihermitian operator and hence $e^{\widehat{T}}$ is not an unitary transformation) results in non-adjoint pairs of right (given by equation (20)) and left eigenvectors,

$$
\langle\tilde{k}|=\langle 0| \widehat{\mathcal{L}}_{k} e^{\widehat{-T}},
$$

associated to the same EOM-CCSD eigenvalue,

$$
\langle 0| \widehat{\mathcal{L}}_{k} e^{-\widehat{T}} \widehat{H}_{\tilde{N}} e^{\widehat{T}}=\Delta E_{k}^{\mathrm{EOM}-\mathrm{CCSD}}\langle 0| \widehat{\mathcal{L}}_{k},
$$

with,

$$
\widehat{\mathcal{L}}_{k}=l_{0}+\sum_{i_{1}, a_{1}} l_{a_{1}}^{i_{1}} \widehat{i}_{1}^{\dagger} \widehat{a}_{1}+\sum_{\substack{a_{1}<a_{2} \\ i_{1}<i_{2}}} l_{a_{1} a_{2}}^{i_{1} i_{2}} \widehat{i}_{1}^{\dagger} \widehat{a}_{1} \widehat{i}_{2}^{\dagger} \widehat{a}_{2}+\ldots
$$

Although the vectors $\langle\tilde{k}|$ and $|k\rangle$ are not adjoint of each other, they satisfy the biorthonormality relation,

$$
\langle\tilde{k} \mid l\rangle=\delta_{k l},
$$

and thus, the multiplication of $\langle 0| \widehat{\mathcal{L}}_{k}$ on the left of equation (22) allows to obtain $\Delta E_{k}^{\mathrm{EOM}-\mathrm{CCSD}}$ with the functional form,

$$
\Delta E_{k}^{\text {EOM-CCSD }}=\left\langle 0\left|\widehat{\mathcal{L}}_{k} e^{-\widehat{T}} \widehat{H}_{\tilde{N}} e^{\widehat{T}} \widehat{\mathcal{R}}_{k}\right| 0\right\rangle .
$$

Finally, the energy of the $k$-th excited state, $E_{k}$, is given by

$$
E_{k}^{\mathrm{EOM}-\mathrm{CCSD}}=E_{\mathrm{GS}}^{\mathrm{CCSD}}+\Delta E_{k}^{\mathrm{EOM}-\mathrm{CCSD}},
$$


in which $E_{\mathrm{GS}}^{\mathrm{CCSD}}$ represents the CCSD ground state energy. Hence, molecular properties of excited states, i.e., derivatives of $E_{k}^{\mathrm{EOM}-\mathrm{CCSD}}$ with respect to a perturbation $x$ (e.g., an external electric or magnetic field or a nuclear coordinate), have a component from (i) the GS ${ }^{[36]}$ and another from (ii) the excitation energy. The obtention of CC ground state properties ${ }^{[37]}$ as well as the derivatives of $\Delta E_{k}^{\mathrm{EOM}-\mathrm{CCSD}[35]}$ are well established. The derivatives of both $E_{\mathrm{GS}}^{\mathrm{CCSD}}$ and $\Delta E_{k}^{\mathrm{EOM}-\mathrm{CCSD}}$ with respect to $x$ lead to the one- $\left(\mathbf{D}^{k}\right)$ and two-electron $\left(\mathbf{d}^{k}\right)$ matrices of the $k$-th excited state whose elements can be written as, ${ }^{[35]}$

$$
\begin{aligned}
D_{p q}^{k} & =\left\langle 0\left|\widehat{\mathcal{L}}^{k} e^{-\widehat{T}} \widehat{p}^{\dagger} \widehat{q} e^{\widehat{T}} \widehat{\mathcal{R}}^{k}\right| 0\right\rangle+\left\langle 0\left|\widehat{\mathcal{Z}}^{k} e^{-\widehat{T}} \widehat{p}^{\dagger} \widehat{q} e^{\widehat{T}}\right| 0\right\rangle, \\
d_{p q r s}^{k} & =\left\langle 0\left|\widehat{\mathcal{L}}^{k} e^{-\widehat{T}} \widehat{p}^{\dagger} \widehat{r}^{\dagger} \widehat{q} \widehat{s} e^{\widehat{T}} \widehat{\mathcal{R}}\right| 0\right\rangle+\left\langle 0\left|\widehat{\mathcal{Z}}^{k} e^{-\widehat{T}} \widehat{p}^{\dagger} \widehat{r}^{\dagger} \widehat{q} \widehat{s} e^{\widehat{T}}\right| 0\right\rangle,
\end{aligned}
$$

where $\widehat{\mathcal{Z}}^{k}$ is a deexcitation operator which is described in detail in reference [35]. The matrix elements in expressions (29) and (30) together with formulae (10) and (11) yield the reduced first order density matrix and the pair density of the $k$-th excited state, the necessary scalar fields to perform the IQA analysis for this electronic state. Once performed the IQA division for the ground and the $k$-th excited state, it is straightforward to partition the corresponding EOM-CCSD excitation energy,

$$
\begin{aligned}
\Delta E_{k}^{\mathrm{EOM}-\mathrm{CCSD}} & =E_{k}^{\mathrm{EOM}-\mathrm{CCSD}}-E_{\mathrm{GS}}^{\mathrm{CCSD}} \\
& =\sum_{\mathrm{A}} \Delta E_{\mathrm{net}}^{\mathrm{A}}+\sum_{\mathrm{A}>\mathrm{B}} \Delta E_{\mathrm{int}}^{\mathrm{AB}},
\end{aligned}
$$

into intra-atomic and interaction contributions. Likewise to the ground state case, the interaction energy components can be divided in classical and exchange-correlation terms according to Eq. (6).

\section{Computational details}

We performed a detailed analysis of the excitation energy partition of two different kinds of systems. First, we performed the dissection of $\Delta E$ for low energy excited states of $\mathrm{N}_{2}, \mathrm{CO}$, 
$\mathrm{H}_{2} \mathrm{O}, \mathrm{C}_{2} \mathrm{H}_{4}$ and $\mathrm{CH}_{4}$. Second, we carried out the IQA/EOM-CCSD analysis of the helium dimer particularly in those electronic states which lead to the formation of the He excimer. We considered two ways to determine the geometry of the molecules of interest, (i) to take the geometry directly from the literature that report the addressed molecular excitation energies as done for $\mathrm{C}_{2} \mathrm{H}_{4}, \mathrm{H}_{2} \mathrm{O}$ and $\mathrm{CH}_{4}$ or (ii) to perform geometry optimisations with the CSSD/Sadlej-pVTZ and CCSD/Sadlej+ approximations for CO and $\mathrm{N}_{2}$ respectively as implemented in the Psi4 ${ }^{[38]}$ code. The Electronic Supporting Information (Esi) reports the Cartesian coordinates of the examined molecules and the way in which we determined them. Concerning the He dimer, we computed potential energy curves for the GS and the electronic systems related with the generation of the helium excimer with the CCSD/d-augcc-pVDZ and EOM-CCSD/d-aug-cc-pVDZ approximation respectively. Once the geometry of the systems under consideration was established, we performed the excitation energies partition for low energy excited states of these systems according to the IQA/EOM-CCSD method. For this purpose, we chose the medium-size polarised basis sets of Sadlej ${ }^{[39]}$ to examine the molecular systems of interest. These atomic basis sets were designed for high-level correlated calculations of molecular properties. We used the Sadlej-pVTZ atomic basis set for $\mathrm{H}_{2} \mathrm{O}$, CO, $\mathrm{CH}_{4}$ and $\mathrm{C}_{2} \mathrm{H}_{4}$ species and Sadlej+ for the $\mathrm{N}_{2}$ molecule. We performed the IQA/EOM-CCSD method in the helium dimer with the basis d-aug-cc-pVDZ. This choice of the atomic basis set is accordance to data reported in the previous literature ${ }^{[40-43]}$. The first step is to calculate the energy of the GS and the vertical excited states for each molecule using CCSD and EOM-CCSD theories respectively. We have employed the package MrCC ${ }^{[44,45]}$ for this purpose, since it allows us to print the EOM-CCSD and CCSD one- and two-electrons density matrices in the basis of Hartree-Fock canonical orbitals. The resulting first and second order density matrices are used by different MrCC modules to calculate molecular properties, e.g., analytic gradients. As stated in section 2, these matrix elements are necessary to carry out the IQA energy partition scheme. We used our in-house PROMOLDEN ${ }^{[46,47]}$ code to perform the QTAIM and IQA calculations. This package computes the atomic surfaces of the QTAIM atoms and integrates the IQA terms defined in subsection 2.1. The input file for PROMOLDEN makes reference to a WFN file that is built from Hartree-Fock canonical orbitals and the ma- 
trix elements of the one- and two particles density functions defined in equations (29) and (30) for the ground and the investigated excited states. The total Promolden excitation energy reported in this article is the sum given by the RHS of equation (31). The numerical integrations of the monoelectronic and bi-electronic energetic terms defined in subsection 2.1 were performed using $\beta$-spheres for all atoms, with radio fixed at values between $0.1 a_{0}$ to $0.3 a_{0}$. Inside the $\beta$-spheres, Lebedev angular quadratures with 3074 points and 451 points Gauss-Chebyshev mapped radial grids were used. The angular momentum number $(L)$ of the bipolar expansion employed to obtain the classic and exchange-correlation interaction energy terms was truncated at $L=8$. Outside the $\beta$-spheres, extended 5810-point Lebedev and 551-mapped radial point trapezoidal quadratures were used, with the $L$ expansions truncated at $L=10$. The accumulated experience to date within the IQA method has shown that total energies reconstructed as the sum of the net energies $\left(E_{\text {net }}^{A}\right)$ and interaction energies $\left(E_{\text {int }}^{A B}\right)$

differ in less than $1.0 \mathrm{kcal} \mathrm{mol}^{-1}$ from those of the parent electronic structure code used to obtain the wavefunction. We expect that each computed interaction is considerably more accurate than this figure. It is worth noting that errors in numerical IQA integrations stem from limitations in the quadratures used, approaching the exact value from above or below and thus tending to accumulation, not cancellation.

\section{Results and discussion}

We proceed now to test and illustrate the IQA/EOM-CCSD partition of the excitation energy. We focus on how we can get valuable information about the nature of excited states by means of this real space analysis of electronic wave functions. Before considering in detail the electronically excited species that we have chosen to exemplify the use of the IQA/EOM-CCSD method, we point out that this partition of excitation energies is only meaningful when it is applied in systems for which the EOM-CCSD approximation provides an adequate description. In other words, for molecules and molecular clusters in which (i) their electronic ground states do not present a substantial multiconfigurational character and (ii) their excitation processes are dominated by single excitations. On the other hand, given the complexity of the IQA integrations to partition excitation energies, the IQA/EOM-CCSD is only feasible for Fock 
spaces comprised by a few hundreds of spatial molecular orbitals.

\subsection{Dinitrogen}

The two bottom lines of Table 1 report the EOM-CCSD excitation energies and those obtained by the sum of all the IQA components calculated with PROMOLDEN (RHS of Equation (31)) for the considered low energy excited states of $\mathrm{N}_{2}$. The comparison of these results shows errors in the IQA excitation energy partition that range from $0.05 \mathrm{eV}$ to $0.06 \mathrm{eV}$. Another indicator of the accuracy of the IQA calculation is the fact that the sum of QTAIM atomic charges, in the investigated $\mathrm{N}_{2}$ states is not larger than $0.0037 e$. The overall accuracy is within the expected numerical integration error of the method, hence showing that the IQA/EOMCCSD partition suitably divides the excitation energy of the addressed $\mathrm{N}_{2}$ states. Table 1 also gathers the results for the IQA/EOM-CCSD partition of the excitation energy as well as the changes in QTAIM delocalisation indexes due to photoexcitation.

Several facts stand out. First, the net energy of the $\mathrm{N}$ atom is clearly an increasing function of the excitation energy as shown in Figure 1. This observation implies that the nitrogen basins in the examined excited states of $\mathrm{N}_{2}$ display a considerable intra-atomic reorganization that leads to a considerable increase in the relative value of the atomic net energy. Such rise in net energies will be referred as deformation energy hereafter. The interaction energy components are also interesting. In the $1^{1} \Pi_{g}, 1^{1} \Sigma_{u}^{-}$and $1^{1} \Delta_{u}$ states, the exchange-correlation energy $E_{\mathrm{xc}}$ becomes less stabilising and the delocalisation index (i.e. the number of shared electrons) decreases. In other words, the covalency of the interaction is hampered in these excited states. Electrons localise ${ }^{2}$ in the atomic basins and, concomitantly, the electrostatic repulsion between the atomic densities $\left(E_{\text {class }}\right)$ increases. These results are in agreement with a standard picture in which "antibonding" functions become populated upon photoexcitation. Notice that the ground state DI differs significantly from three as specified in the footnote of Table 1. This observation is a well known effect of electron correlation, which effectively populates virtual,

\footnotetext{
${ }^{2}$ When electrons are shared between two basins A and B, it is said that they delocalise between the corresponding atoms. Because electrons are less available for covalent bonding after photoexcitation as evidenced by the reduction of DIs in Table 1, it follows that electrons are more localised within the basins $\mathrm{A}$ and B.
} 
Table 1: Change of delocalisation indexes (in atomic units) due to photoexcitation for the investigated excited states of $\mathrm{N}_{2}$ in the CCSD/Sadlej-plus optimised geometry. We also present the IQA partition of the excitation energy $(\mathrm{eV})$ in net as well as classical and exchange-correlation inter-atomic components, and the comparison between the sum of these terms and the results obtained directly from EOM-CCSD calculations. ${ }^{\dagger}$

\begin{tabular}{|c|c|c|c|c|c|}
\hline State & $1^{1} \Pi_{g}$ & $1^{1} \Sigma_{u}^{-}$ & $1^{1} \Delta_{u}$ & $1^{1} \Pi_{u}$ & $2{ }^{1} \Pi_{u}$ \\
\hline$\Delta \mathrm{DI}(\mathrm{N}, \mathrm{N})$ & -0.124 & -0.133 & -0.086 & 0.274 & 0.255 \\
\hline \multicolumn{6}{|c|}{ IQA excitation energy partition } \\
\hline$\Delta E_{\text {class }}^{\mathrm{N}, \mathrm{N}}$ & 0.82 & 0.66 & 0.69 & 2.77 & 2.85 \\
\hline$\Delta E_{\mathrm{xc}}^{\mathrm{N}, \mathrm{N}}$ & 1.56 & 1.60 & 1.18 & 0.43 & 0.33 \\
\hline $2 \Delta E_{\text {net }}$ & 6.88 & 7.60 & 8.41 & 9.48 & 10.16 \\
\hline$\Delta E$ & 9.26 & 9.86 & 10.28 & 12.68 & 13.34 \\
\hline$\Delta E_{\mathrm{MRCC}}$ & 9.32 & 9.91 & 10.34 & 12.74 & 13.39 \\
\hline \multicolumn{6}{|c|}{$\begin{array}{l}{ }^{\dagger} \text { The corresponding values (in atomic units) for the } \mathrm{N}_{2} \text { ground state are } \mathrm{DI}(\mathrm{N}, \mathrm{N})=2.048, E_{\mathrm{class}}^{\mathrm{N}, \mathrm{N}}=0.224, E_{\mathrm{xc}}^{\mathrm{N}, \mathrm{N}}=-0.700 \text { anc } \\
E_{\mathrm{net}}^{\mathrm{N}}=-54.407 \text {. }\end{array}$} \\
\hline
\end{tabular}

The $1,2{ }^{1} \Pi_{u}$ excitations break the above rule. Both display large destabilising $E_{\text {class }}$ as well as increasing DI, although the latter is not accompanied by a stabilising $E_{\mathrm{xc}}$. This negative correlation between DI and $E_{\mathrm{xc}}$ can be rationalised by taking into account that the excitation changes the compact nature of shared electrons in the ground state into a more diffuse one $\left(\Delta E_{\text {class }}>0\right)$ and less energetically stabilising $\left(\Delta E_{\mathrm{xc}}>0\right)$. The increase of the DI over the GS value is likely related to a behaviour that has been previously described. ${ }^{[49]}$ It emerges whenever zwitterionic or ionic resonance structures

$$
{ }^{-}: \ddot{\mathrm{N}}=\mathrm{N}:^{+} \longleftrightarrow{ }^{+}: \mathrm{N}=\ddot{\mathrm{N}}:^{-}
$$

become important. It may be shown that in the case of a two-centre, two-electron bond, e.g. $\mathrm{H}_{2}$

$$
{ }^{-}: \mathrm{H} \mathrm{H} \mathrm{H}^{+} \longleftrightarrow \mathrm{H}-\mathrm{H} \longleftrightarrow{ }^{+} \mathrm{H} \mathrm{H}:^{-}
$$

the pure ionic resonance in which the two electrons are either found on one center or on the other (with probability $1 / 2$ ) leads to a value of $\mathrm{DI}(\mathrm{A}, \mathrm{B})=2$. This result is evidenced via the relation between the delocalisation index and the covariance of the two-center joint 


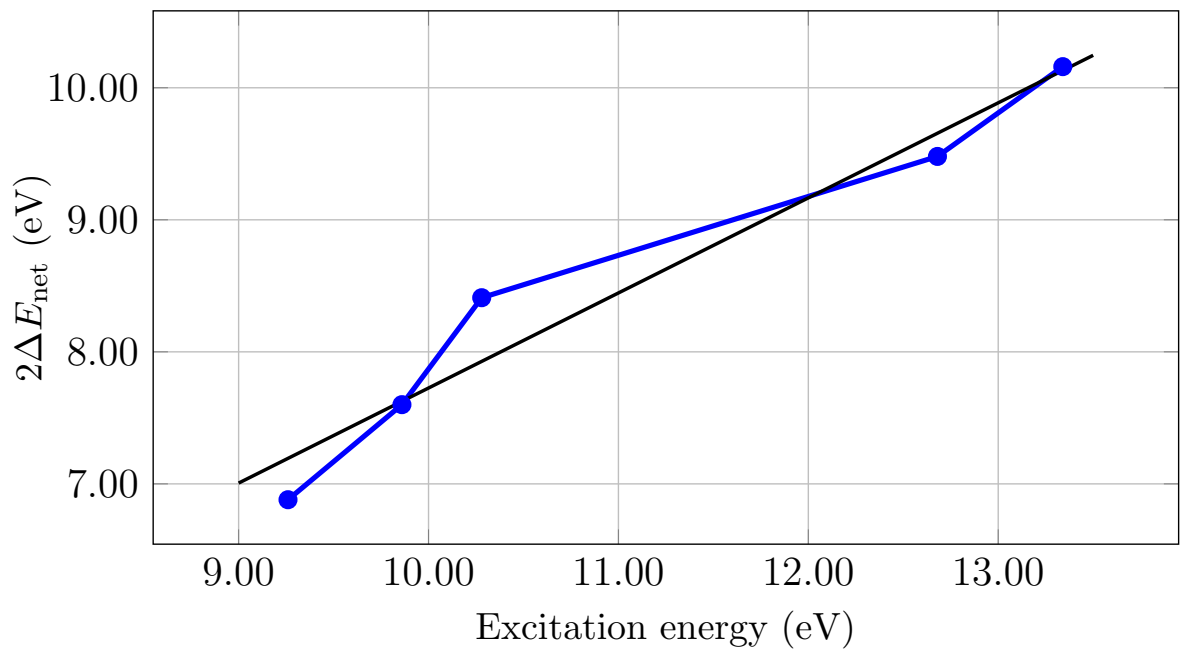

Figure 1: Correlation between net and the excitation energies (both in $\mathrm{eV}$ ) in the electronic excited states of $\mathrm{N}_{2}$ reported in Table 1 . The value of the square of the linear regression coefficient is 0.950 .

probability distribution of electron populations, $\mathrm{DI}(\mathrm{A}, \mathrm{B})=-2 \operatorname{cov}\left(n_{\mathrm{A}}, n_{\mathrm{B}}\right)$. A more complete characterisation of the real space features of the $1,2^{1} \Pi_{u}$ states is possible in terms of electron distribution functions (EDF), ${ }^{[50]}$ and natural adaptive orbitals, ${ }^{[51]}$ although this endeavour is out of the scope of this research.

\subsection{Carbon monoxide}

We show the IQA partition of the vertical excitation energies of a few low-energy valence singlet states of carbon monoxide in Table 2. The EOM-CCSD excitation energies computed with the MRCC program are almost completely recovered by the sum of IQA components with errors not larger than $0.05 \mathrm{eV}$, a condition which provides a measure of the quality of the IQA integration method in Promolden. We also used the total integrated charge (the sum of all atomic charges) to assess the performance of the integration method in the IQA energy decomposition technique. In all the examined excited states of $\mathrm{CO}$, we registered a maximum total charge of $0.011 e$, an error which lies in the expected accuracy of the code.

Concerning representative IQA/QTAIM indicators for the investigated excitation processes, we note that there is not a clear trend concerning the changes of QTAIM charges with respect to excitation energies as revealed by the alternating signs of $\Delta q^{\mathrm{C}}$ and $\Delta q^{\mathrm{O}}$ throughout Table 2 . These changes in the atomic charges are as expected accompanied by relevant 
Table 2: Changes of delocalisation indexes and atomic charges (both in atomic units) due to photoexcitation processes which conduce to the investigated low energy valence singlet excited states of CO in the CCSD optimised geometry. We also report the IQA division of the excitation energy (in eV) in net, classical and exchange-correlation terms as well as the comparison between the total IQA excitation energies $(\Delta E)$ and the corresponding value computed directly from EOM-CCSD calculations $\left(\Delta E_{\mathrm{MrCC}}\right) . \ddagger$

\begin{tabular}{|c|c|c|c|c|c|c|c|}
\hline State & $1^{1} \Pi$ & $1^{1} \Sigma^{-}$ & $2^{1} \Sigma^{+}$ & $3^{1} \Sigma^{+}$ & $2^{1} \Sigma^{-}$ & $4^{1} \Sigma^{+}$ & $3^{1} \Sigma^{-}$ \\
\hline$\Delta \mathrm{DI}(\mathrm{C}, \mathrm{O})$ & 0.196 & -0.246 & -0.256 & 0.525 & 0.363 & 0.024 & -0.054 \\
\hline$\Delta q^{\mathrm{C}}$ & -0.289 & -0.442 & -0.418 & 0.000 & -0.183 & 0.103 & 0.153 \\
\hline$\Delta q^{\mathrm{O}}$ & 0.291 & 0.443 & 0.419 & 0.007 & 0.195 & -0.102 & -0.151 \\
\hline \multicolumn{8}{|c|}{ IQA division of the excitation energy } \\
\hline$\Delta E_{\text {class }}^{\mathrm{C}, \mathrm{O}}$ & 14.48 & 15.18 & 14.64 & 4.91 & 5.38 & 5.79 & 5.46 \\
\hline$\Delta E_{\mathrm{xc}}^{\mathrm{C}, \mathrm{O}}$ & -1.47 & 1.99 & 2.03 & -1.39 & -1.70 & 1.66 & 1.90 \\
\hline$\Delta E_{\text {net }}^{\mathrm{C}}$ & -1.31 & -5.65 & -5.16 & 8.93 & 10.69 & 5.90 & 5.83 \\
\hline$\Delta E_{\text {net }}^{\mathrm{O}}$ & -3.00 & -1.48 & -1.37 & 0.93 & -0.68 & 1.08 & 1.29 \\
\hline$\Delta E$ & 8.70 & 10.04 & 10.14 & 13.39 & 13.69 & 14.44 & 14.49 \\
\hline$\Delta E_{\mathrm{MRCC}}$ & 8.68 & 10.01 & 10.13 & 13.41 & 13.74 & 14.44 & 14.51 \\
\hline
\end{tabular}

modifications in classical terms. On the other hand, and not surprisingly, the delocalisation index runs rather parallel to $E_{\mathrm{xc}}$, becoming more stabilising for states $1{ }^{1} \Pi, 3{ }^{1} \Sigma^{+}$and $2^{1} \Sigma^{-}$ whereas the rest of excited states in Table 2 exhibit the opposite behaviour.

We stress that two opposite behaviours are found in (i) $\mathrm{N}_{2}$, a covalently stabilised molecule, and (ii) CO, a species whose GS is more stabilised by $E_{\text {class }}^{\mathrm{C}, \mathrm{O}}\left(-1.244 E_{\mathrm{h}}\right)$ than it is by $E_{\mathrm{xc}}^{\mathrm{C}, \mathrm{O}}$ $\left(-0.441 E_{\mathrm{h}}\right)$ as can be noted in the footnote of Table 2 . The ground state in nitrogen minimises the classical repulsion among the atoms and the net energies of their atoms, $E_{\text {class }}$ and $E_{\text {net }}$ respectively. On the contrary, the GS in CO displays the largest classical attraction. Although $E_{\text {class }}^{\mathrm{C}, \mathrm{O}}$ is attractive for all the investigated electronic states of carbon monoxide, every value of $\Delta E_{\text {class }}^{\mathrm{C}, \mathrm{O}}$ is positive in Table 2. Interestingly, there are states of $\mathrm{CO}$ with larger charge transfers than that found in the GS (i.e. with $\Delta q^{\mathrm{O}}<0$ and $\Delta q^{\mathrm{C}}>0$ so that the magnitudes of the atomic charges are larger than those in the ground state) which do not provide more stabilising $E_{\text {class }}$ values, i.e., $4^{1} \Sigma^{+}$and $3^{1} \Sigma^{-}$. These results shows the importance of the 
changes of the rest of atomic multipole moments in the system.

The behaviour of the net energies is also to be noticed. The range of variation of $E_{\text {net }}$ in the $\mathrm{C}$ atom is much larger than it is in the $\mathrm{O}$ atom. Concerning IQA net energies, we observe that in most cases (except for $\left.1^{1} \Pi\right)\left|\Delta E_{\text {net }}^{\mathrm{C}}\right|>\left|\Delta E_{\text {net }}^{\mathrm{O}}\right|$. We note that apart from the $2^{1} \Sigma^{-}$state, $\Delta q^{\mathrm{C}}$ and $\Delta E_{\text {net }}^{\mathrm{C}}$ have the same sign, which means that the net energy of carbon decreases with the number of electrons within this atom. The oxygen shows the opposite behaviour, a condition which suggest a saturation of electron charge in this atom. Positive IQA deformation energies in negatively charged quantum atoms are commonplace. These energies are associated to the multipolar distortion of largely polarisable QTAIM basins with an excess of electron charge. We also observed that the exchange-correlation can substantially stabilise the CO interaction for example in the $2^{1} \Sigma^{-}$state which presents a considerable deformation of the carbon atom as evidenced by a considerable positive value of $\Delta E_{\text {net }}^{\mathrm{C}}$. The anomalously high net energy of $\mathrm{C}$ in this state must thus be related to an intra-atomic promotion that allows for further electron delocalisation.

The landscape pictured here shows the complexity of energy redistribution in excited states when charge transfer is important. If a GS is very polarised, as it is the case in $\mathrm{CO}$, photoexcitation may give rise to electronic redistribution that increase or decrease the atomic charges. Since excitation usually leads to stronger asphericity of the electron density, monopolar character tends to be substituted by multipolar components, which decay faster with distance and are therefore less efficient in the electrostatic stabilisation. In the CO case, the interaction energy becomes less stabilising, i.e., $\Delta E_{\mathrm{int}}^{\mathrm{C}, \mathrm{O}}=\Delta E_{\mathrm{xc}}^{\mathrm{C}, \mathrm{O}}+E_{\text {class }}^{\mathrm{C}, \mathrm{O}}>0$ in all the examined excited states of CO. This weakened CO interaction is not balanced by the more negative net energies due to smaller atomic charges. In the lowest lying $1^{1} \Pi$ states, for instance, the excited atoms lie $4.31 \mathrm{eV}$ below those in the GS due to their smaller charge, and the $\mathrm{CO}$ bond is also considerably more covalent. However, $E_{\text {class }}$ is $14.45 \mathrm{eV}$ less negative in the excited state, a condition which indicates the above-mentioned stabilising electron distributions which occurs in the ground state of CO. 


\subsection{Water}

The excited states of the water molecule have been extensively studied, ${ }^{[41,42]}$ and therefore, they offer a good opportunity to illustrate the IQA/EOM-CCSD method. We present in Table 3 the EOM-CCSD excitation energies computed with the programs MrCC and PROMOLDEN for the lowest energy singlet valence states of $\mathrm{H}_{2} \mathrm{O}$. Once again, the IQA energy partition recovers the total excitation energy of the electronic structure calculation with errors that are smaller than $0.02 \mathrm{eV}$. In the same way, the accuracy of the QTAIM/IQA integration is again tested with the sum of the QTAIM atomic charges of the examined electronic excited states of the $\mathrm{H}_{2} \mathrm{O}$ molecule. We found that the total charge is, in any case, not larger than $0.0013 e$.

Concerning the QTAIM properties and the IQA energy decomposition contributions of the investigated electronic states of $\mathrm{H}_{2} \mathrm{O}$ (Table 3 ), these parameters have a somewhat erratic behaviour in the considered interval of excitation energies. This absence of correlation between the IQA and QTAIM indexes with the excitation energy indicates that the nature of the excited state depends strongly not only on the energy of the absorbed photon but also on the nature of the occupied and virtual orbitals involved in the process.

Table 3 shows that the polarity of the $\mathrm{O}-\mathrm{H}$ interaction varies considerably among the studied excited states. We emphasise that the charges of the two $\mathrm{H}$ atoms must be identical in all the electronic states considered. ${ }^{3}$ In some states, particularly in $3{ }^{1} \mathrm{~A}_{1}, 2{ }^{1} \mathrm{~B}_{1}$ and $2{ }^{1} \mathrm{~B}_{2}$, the charge of the oxygen atom is considerably more negative than it is in the GS, leading to an increase in the stabilising character of $E_{\text {class }}^{\mathrm{O}, \mathrm{H}}$ and to a concomitant decrease of the covalency of the interaction. In the last two-mentioned states, the total $\mathrm{O}-\mathrm{H}$ interaction is slightly larger in magnitude than the corresponding value in the GS. This increased polarity is obtained at the expense of a clear increase of the net energy of the atoms within the $\mathrm{H}_{2} \mathrm{O}$ molecule, as revealed by the associated $\Delta E_{\text {net }}^{\mathrm{O}}$ and $\Delta E_{\text {net }}^{\mathrm{H}}$ values, which are among the most destabilising components within the examined excited states of $\mathrm{H}_{2} \mathrm{O}$. The $\mathrm{H} \cdots \mathrm{H}$ interaction is interesting in itself. The classical $\mathrm{H} \cdots \mathrm{H}$ interaction is always positive because the two hydrogen atoms and their associated electron densities are symmetrically equivalent. Two states, $1{ }^{1} \mathrm{~A}_{2}$

\footnotetext{
${ }^{3}$ Because $\mathcal{C}_{2 v}$ is an Abelian group, all the examined electron and pair densities are completely symmetric ${ }^{[52]}$
} 
Table 3: Changes of atomic charges and delocalisation indexes (in atomic units) for $\mathrm{H}_{2} \mathrm{O}$ as a consequence of photoexcitations which results in the lowest singlet excited states of the water monomer. We also present (in $\mathrm{eV}$ ) the IQA partition of the excitation energy in net, classical and exchange-correlation contributions along with the comparison between the excitation energy computed (i) by the sum of the IQA components and (ii) directly from EOM-CCSD calculations. ${ }^{\S}$

\begin{tabular}{|c|c|c|c|c|c|c|c|c|c|}
\hline State & ${ }^{1} \mathrm{~B}_{2}$ & $1^{1} \mathrm{~A}_{2}$ & $2{ }^{1} \mathrm{~A}_{1}$ & $1^{1} \mathrm{~B}_{1}$ & $2{ }^{1} \mathrm{~A}_{2}$ & $2^{1} \mathrm{~B}_{2}$ & $2^{1} \mathrm{~B}_{1}$ & $3^{1} \mathrm{~B}_{1}$ & $3^{1} \mathrm{~A}_{1}$ \\
\hline$\Delta \mathrm{DI}(\mathrm{H}, \mathrm{H})$ & 0.127 & 0.369 & 0.075 & 0.353 & 0.051 & -0.012 & -0.008 & 0.123 & 0.026 \\
\hline$\Delta \mathrm{DI}(\mathrm{O}, \mathrm{H})$ & 0.031 & -0.126 & 0.016 & -0.145 & 0.038 & -0.186 & -0.098 & -0.051 & -0.027 \\
\hline$\Delta q^{\mathrm{O}}$ & 0.282 & 0.599 & 0.107 & 0.473 & 0.192 & -0.328 & -0.208 & 0.068 & -0.065 \\
\hline$\Delta q^{\mathrm{H}}$ & -0.141 & -0.300 & -0.054 & -0.237 & -0.096 & 0.164 & 0.103 & -0.034 & 0.032 \\
\hline \multicolumn{10}{|c|}{ IQA excitation energy partition } \\
\hline$\Delta E_{\text {class }}^{\mathrm{H}, \mathrm{H}}$ & -0.29 & -0.65 & 0.42 & -0.05 & -0.60 & 1.94 & 1.28 & 0.72 & 1.29 \\
\hline$\Delta E_{\mathrm{xc}}^{\mathrm{H}, \mathrm{H}}$ & -0.23 & -0.52 & -0.12 & -0.48 & -0.14 & 0.04 & 0.03 & -0.24 & -0.01 \\
\hline $2 \Delta E_{\text {class }}^{\mathrm{O}, \mathrm{H}}$ & 4.36 & 6.90 & 1.02 & 4.30 & 7.25 & -4.53 & -2.55 & 3.05 & -2.44 \\
\hline $2 \Delta E_{\mathrm{xc}}^{\mathrm{O}, \mathrm{H}}$ & 1.56 & 2.59 & 2.31 & 3.44 & 1.14 & 3.29 & 2.23 & 3.40 & 2.81 \\
\hline$\Delta E_{\mathrm{net}}^{\mathrm{O}}$ & 1.25 & -0.14 & 3.47 & 1.53 & 2.54 & 7.64 & 8.40 & 2.40 & 8.38 \\
\hline $2 \Delta E_{\mathrm{net}}^{\mathrm{H}}$ & 0.72 & 0.96 & 2.71 & 2.82 & 1.51 & 4.90 & 4.18 & 4.46 & 5.51 \\
\hline$\Delta E$ & 7.37 & 9.15 & 9.81 & 11.56 & 11.70 & 13.28 & 13.57 & 13.79 & 15.54 \\
\hline$\Delta E_{\mathrm{MRCC}}$ & 7.38 & 9.15 & 9.81 & 11.55 & 11.71 & 13.28 & 13.57 & 13.80 & 15.53 \\
\hline
\end{tabular}

and $1{ }^{1} \mathrm{~B}_{1}$ display very large values of $\mathrm{DI}(\mathrm{H}, \mathrm{H})$ up to 0.390 in the former state. This large delocalisation indexes are accompanied by non-negligible $E_{\mathrm{xc}}^{\mathrm{H}, \mathrm{H}}$ components, of about $-0.60 \mathrm{eV}$ $\approx-13.8 \mathrm{kcal} \mathrm{mol}^{-1}$. These two states possess the two smallest QTAIM charges, showing that the overall polarity of the molecule has clearly decreased in consistency with the fact that the corresponding values of $\Delta E_{\text {class }}^{\mathrm{O}, \mathrm{H}}$ are considerably positive. Additionally, the covalency of the $\mathrm{O}-\mathrm{H}$ bond decreases notoriously too, as indicated by the associated values of $\Delta \mathrm{DI}(\mathrm{O}, \mathrm{H})$ and $\Delta E_{\mathrm{xc}}^{\mathrm{O}, \mathrm{H}}$ in Table 3.

Another point that deserves mentioning is that all the examined excited states exhibit positive contributions of $\Delta E_{\mathrm{xc}}^{\mathrm{O}, \mathrm{H}}$. In other words, the GS displays the largest magnitude of all the $E_{\mathrm{xc}}^{\mathrm{O}, \mathrm{H}}$ values, and thus, although, charge transfer has an important role in the stabilisation 
of $\mathrm{H}_{2} \mathrm{O}$, it is actually the $\mathrm{O}-\mathrm{H}$ covalency which drives the energy minimum. In this sense, binding in water has a different behaviour to that in carbon monoxide, in which the GS maximises the electrostatic, not the covalent term. We also point out that the net energies of the $\mathrm{O}$ and $\mathrm{H}$ atoms increase monotonically with the excitation energies within a given irreducible representation of the $\mathcal{C}_{2 v}$ point group of the water molecule.

As opposed to the behaviour previously described for $\mathrm{N}_{2}$, the sum of the atomic net energy is not an increasing function of the total electronic energy of the system. These results indicate that in some instances the energy of the photon leads to a considerable change in the chemical bonding scenario of the $\mathrm{H}_{2} \mathrm{O}$ molecule and the energy of the absorbed

photon is stored in its chemical bonds, e.g., concerning the $2{ }^{1} \mathrm{~A}_{2}$ state, we have $2 \Delta E_{\text {int }}^{\mathrm{O} H}=$ $2\left(\Delta E_{\text {class }}^{\mathrm{O}, \mathrm{H}}+\Delta E_{\mathrm{xc}}^{\mathrm{O}, \mathrm{H}}\right)=8.39 \mathrm{eV}$ which represents more than $70 \%$ of the excitation energy We observed, however, the opposite situation for the $3{ }^{1} \mathrm{~A}_{1}$ state, i.e., the energy of the absorbed photon is mainly collected in the oxygen and hydrogen atoms mainly, $\Delta E_{\text {net }}^{\mathrm{O}}+2 \Delta E_{\text {net }}^{\mathrm{H}}=$ $13.89 \mathrm{eV}$ an amount that adds up to $89 \%$ of the excitation energy. In the same regard, we observe a few cases, like the $1^{1} \mathrm{~A}_{2}$ state, for which the excited water molecule behaves very differently from its GS counterpart: smaller charge separation clearly weakens the $\mathrm{O}-\mathrm{H}$ interaction (both electrostatically and covalently) and leads to a much larger $\mathrm{H} \cdots \mathrm{H}$ covalency. This kind of information provided by the IQA decomposition might be very useful in the rational design of specific excited state reactivity.

\subsection{Ethylene}

The ethylene molecule is the simplest archetype to study electronic photoexcitations which involve carbon-carbon multiple bonds in organic molecules. The electrons that form the double bond in $\mathrm{C}_{2} \mathrm{H}_{4}$ have many different possibilities of photoexcitation. Table 4 shows the

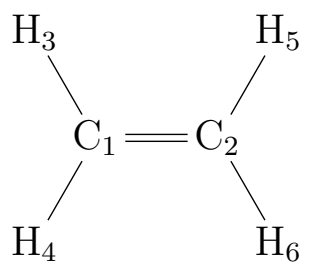

Figure 2: Atomic labelling used in the discussion of the partition of the investigated excitation energies of the ethylene molecule. 
changes in QTAIM charges and delocalisation indexes as well as the IQA excitation energy partition associated to some low energy valence and Rydberg states of the ethylene molecule. We point out that all the excited states of ethylene examined herein, except for the $1{ }^{1} \mathrm{~B}_{1 u}$ state which is the only one that corresponds to a pure valence transition, have a very large Rydberg character $^{[40]}$. Due to the transition of the electrons to regions which are far away from the nuclei, the IQA integration for Rydberg states presents a few problems. Nevertheless, and likewise to the previously discussed molecules, the PROMOLDEN code recovers the excitation energy in the IQA integration with relative accuracy, with errors in the range from $0.01 \mathrm{eV}$ to $0.06 \mathrm{eV}$. We were also able to obtain acceptable results for the integration of charge, the corresponding errors being in the interval $0.003 e$ to $0.017 e$.

Concerning the results reported in Table 4, we first point out that $\mathcal{D}_{2 h}$ is an Abelian group, and hence, every density function in ethylene is completely symmetrical, as it is the case in the previously discussed electronic states of $\mathrm{H}_{2} \mathrm{O}$. We observe that photoexcitation reduces in most cases the number of shared electrons for the $\mathrm{C}=\mathrm{C}$ and $\mathrm{C}-\mathrm{H}$ covalent bonds as measured by the delocalisation indices between these two pairs of atoms. The reduction in the number of these delocalised electrons is accompanied by positive values of $\Delta E_{\mathrm{xc}}^{\mathrm{C}_{1}, \mathrm{C}_{2}}$ and $\Delta E_{\mathrm{xc}}^{\mathrm{C}_{1}, \mathrm{H}_{3}}$ (the atom numbering of ethylene used in this discussion is shown in Figure 1). Hereof, photoexcitation reduces considerably the covalency of the $\mathrm{C}=\mathrm{C}$ bond, a necessary condition for an important part of the reactivity of these chemical bonds in photochemistry, i.e., their rotation that leads to cis-trans isomerisation reactions that might occur in carbon-carbon double bonds ${ }^{[53]}$. We recall herein that photoisomerisations might be followed by other reactions. For example, it is well known that trans cycloalkenes are highly reactive and that these systems can easily suffer subsequent addition reactions. ${ }^{[54]}$ Concerning the classical contributions to the $\mathrm{C}=\mathrm{C}$ and $\mathrm{C}-\mathrm{H}$ chemical bonds, Table 4 shows that $\Delta E_{\text {class }}^{\mathrm{C}_{1}, \mathrm{C}_{2}}>0$ and $\Delta E_{\text {class }}^{\mathrm{C}_{1}, \mathrm{H}_{3}}<0$. Generally, as the classical electrostatic repulsion between the two symmetrically-equivalent carbon atoms increase, the ionic contribution to the carbon-hydrogen bond rises as well, (Figure 3). In most of the investigated excited states of $\mathrm{C}_{2} \mathrm{H}_{4}$, the charge in the carbon basins is negative (Table 11 in the EsI). This is not the case for the $1{ }^{1} \mathrm{~B}_{3 g}$ state wherein photoexcitation changes the polarity of the atoms within the molecule. As expected, the changes in 
Table 4: Changes of delocalisation indices and atomic charges in atomic units for the investigated low-energy valence and Rydberg states of $\mathrm{C}_{2} \mathrm{H}_{4}$. The labels of the atoms in the molecule are shown in Figure 1. We also present the IQA partition of the excitation energy in $\mathrm{eV}$ as well as the comparison between the sum of the IQA components and the excitation energy directly calculated with EOM-CCSD theory. ${ }^{\dagger}$

\begin{tabular}{|c|c|c|c|c|c|}
\hline State & $1^{1} \mathrm{~B}_{1 u}$ & $1^{1} \mathrm{~B}_{3 g}$ & $1^{1} \mathrm{~B}_{3 u}$ & $1^{1} \mathrm{~B}_{2 g}$ & $2{ }^{1} \mathrm{~B}_{1 u}$ \\
\hline$\Delta \mathrm{DI}\left(\mathrm{C}_{1}, \mathrm{C}_{2}\right)$ & -0.041 & -0.067 & 0.355 & -0.067 & 0.133 \\
\hline$\Delta \mathrm{DI}\left(\mathrm{C}_{1}, \mathrm{H}_{3}\right)$ & -0.007 & -0.047 & -0.025 & -0.015 & 0.008 \\
\hline$\Delta \mathrm{DI}\left(\mathrm{H}_{1}, \mathrm{H}_{5}\right)$ & 0.009 & -0.019 & 0.044 & -0.010 & 0.018 \\
\hline$\Delta \mathrm{DI}\left(\mathrm{H}_{3}, \mathrm{H}_{4}\right)$ & 0.034 & 0.080 & -0.006 & 0.064 & -0.011 \\
\hline$\Delta \mathrm{DI}\left(\mathrm{H}_{3}, \mathrm{H}_{5}\right)$ & 0.058 & 0.099 & 0.010 & 0.072 & 0.008 \\
\hline$\Delta \mathrm{DI}\left(\mathrm{H}_{3}, \mathrm{H}_{6}\right)$ & 0.048 & 0.077 & 0.008 & 0.060 & 0.001 \\
\hline$\Delta q^{\mathrm{C}}$ & 0.014 & 0.111 & -0.100 & 0.058 & -0.189 \\
\hline$\Delta q^{\mathrm{H}}$ & -0.003 & -0.053 & 0.051 & -0.025 & 0.095 \\
\hline \multicolumn{6}{|c|}{ IQA excitation energy partition } \\
\hline$\Delta E_{\text {class }}^{\mathrm{C}_{1}, \mathrm{C}_{2}}$ & 0.24 & 0.16 & 0.21 & 0.18 & 0.32 \\
\hline$\Delta E_{\mathrm{xc}}^{\mathrm{C}_{1}, \mathrm{C}_{2}}$ & 0.48 & 0.51 & -0.63 & 0.55 & 0.30 \\
\hline $4 \Delta E_{\text {class }}^{\mathrm{C}_{1}, \mathrm{H}_{3}}$ & -0.67 & -0.51 & -0.61 & -0.49 & -0.99 \\
\hline $4 \Delta E_{\mathrm{xc}}^{\mathrm{C}_{1}, \mathrm{H}_{3}}$ & 1.23 & 1.67 & 0.72 & 1.28 & 0.90 \\
\hline $4 \Delta E_{\text {class }}^{\mathrm{C}_{1}, \mathrm{H}_{5}}$ & 0.52 & 0.60 & 0.04 & 0.58 & 0.19 \\
\hline $4 \Delta E_{\mathrm{xc}}^{\mathrm{C}_{1}, \mathrm{H}_{5}}$ & 0.06 & 0.25 & -0.33 & 0.27 & 0.01 \\
\hline $2 \Delta E_{\text {class }}^{\mathrm{H}_{3}, \mathrm{H}_{4}}$ & 0.40 & 0.36 & 0.26 & 0.43 & 0.56 \\
\hline $2 \Delta E_{\mathrm{xc}}^{\mathrm{H}_{3}, \mathrm{H}_{4}}$ & -0.05 & -0.15 & 0.04 & -0.21 & 0.08 \\
\hline $2 \Delta E_{\text {class }}^{\mathrm{H}_{3}, \mathrm{H}_{5}}$ & 0.19 & 0.21 & 0.14 & 0.20 & 0.29 \\
\hline $2 \Delta E_{\mathrm{xc}}^{\mathrm{H}_{3}, \mathrm{H}_{5}}$ & -0.23 & -0.40 & -0.04 & -0.16 & -0.06 \\
\hline $2 \Delta E_{\text {class }}^{\mathrm{H}_{3}, \mathrm{H}_{6}}$ & 0.12 & 0.09 & 0.12 & 0.14 & 0.25 \\
\hline $2 \Delta E_{\mathrm{xc}}^{\mathrm{H}_{3}, \mathrm{H}_{6}}$ & -0.10 & -0.15 & -0.02 & -0.11 & 0.00 \\
\hline $2 \Delta E_{\text {net }}^{\mathrm{C}}$ & 2.97 & 2.85 & 6.17 & 2.79 & 4.32 \\
\hline $4 \Delta E_{\text {net }}^{\mathrm{H}}$ & 2.16 & 2.46 & 1.93 & 2.52 & 2.97 \\
\hline$\Delta E$ & 7.33 & 7.95 & 8.01 & 7.99 & 9.14 \\
\hline$\Delta E_{\mathrm{MRCC}}$ & 7.30 & 7.97 & 8.00 & 8.01 & 9.08 \\
\hline
\end{tabular}

${ }^{\dagger}$ Results for the GS of $\mathrm{C}_{2} \mathrm{H}_{4}$ in atomic units DI $\left(\mathrm{C}_{1}, \mathrm{C}_{2}\right)=1.335, \mathrm{DI}\left(\mathrm{C}_{1}, \mathrm{H}_{3}\right)=0.802, \mathrm{DI}\left(\mathrm{C}_{1}, \mathrm{H}_{5}\right)=0.059, \mathrm{DI}\left(\mathrm{H}_{3}, \mathrm{H}_{4}\right)=0.050$, $\mathrm{DI}\left(\mathrm{H}_{3}, \mathrm{H}_{5}\right)=0.024, \mathrm{DI}\left(\mathrm{H}_{3}, \mathrm{H}_{6}\right)=0.019, q^{\mathrm{C}}=-0.091, q^{\mathrm{H}}=0.046, E_{\text {net }}^{\mathrm{C}}=-37.612, E_{\text {net }}^{\mathrm{H}}=-0.466, E_{\text {class }}^{\mathrm{C}_{1}, \mathrm{C}_{2}}=0.064, E_{\mathrm{xc}}^{\mathrm{C}_{1}, \mathrm{C}_{2}}$ $=-0.433, E_{\text {class }}^{\mathrm{C}_{1}, \mathrm{H}_{3}}=0.038, E_{\mathrm{xc}}^{\mathrm{C}_{1}, \mathrm{H}_{3}}=-0.258, E_{\text {class }}^{\mathrm{C}_{1}, \mathrm{H}_{5}}=0.002, E_{\mathrm{xc}}^{\mathrm{C}_{1}, \mathrm{H}_{5}}=-0.007, E_{\text {class }}^{\mathrm{H}_{3}, \mathrm{H}_{4}}=0.004, E_{\mathrm{xc}}^{\mathrm{H}_{3}}$ $=0.001, E_{\mathrm{xc}}^{\mathrm{H}_{3}, \mathrm{H}_{5}}=-0.003, E_{\mathrm{class}}^{\mathrm{H}_{3}, \mathrm{H}_{6}}=0.001, E_{\mathrm{xc}}^{\mathrm{H}_{3}, \mathrm{H}_{6}}=-0.001$. 


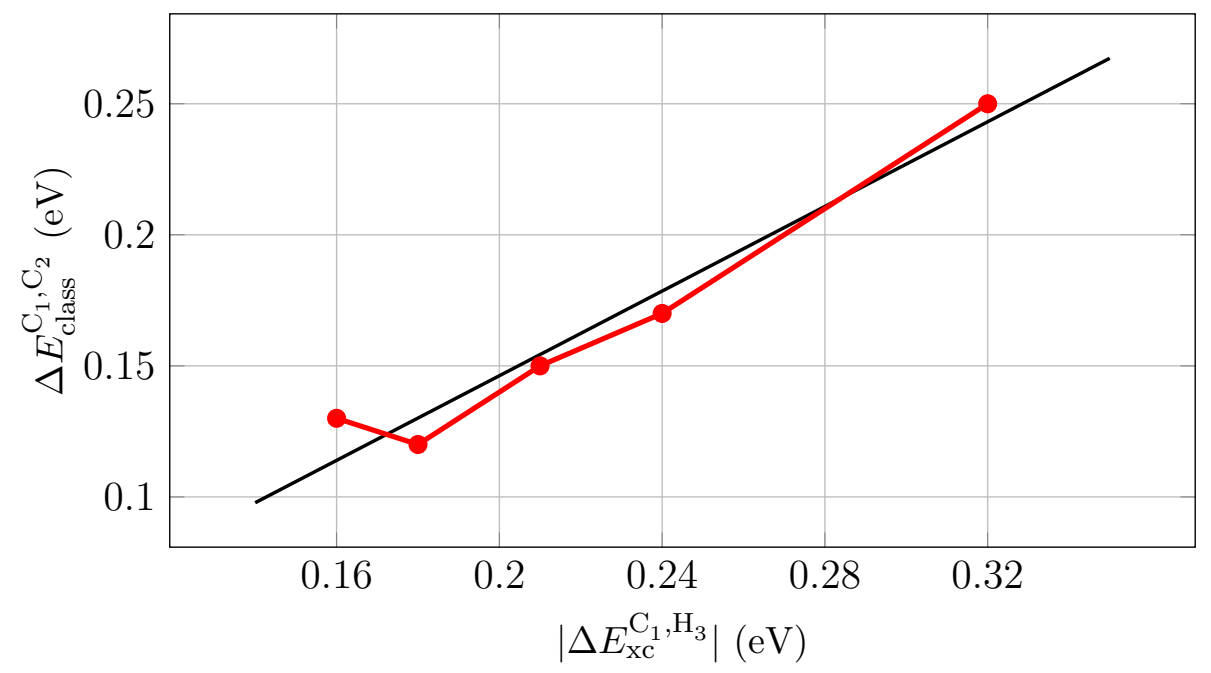

Figure 3: Correlation between $\Delta E_{\text {class }}^{\mathrm{C}_{1}, \mathrm{C}_{2}}$ and $\left|\Delta E_{\mathrm{xc}}^{\mathrm{C}_{1}, \mathrm{H}_{3}}\right|$ for the investigated excited states of $\mathrm{C}_{2} \mathrm{H}_{4}\left(R^{2}=\right.$ $0.963)$.

$\mathrm{C} \cdots \mathrm{H}$ and $\mathrm{H} \cdots \mathrm{H}$ interactions are more subtle. Similarly to the case of water, photoexcitation increases the covalent character of the later type of interactions. Notwithstanding the relevant changes in the chemical bonding scenario of the excited states of $\mathrm{C}_{2} \mathrm{H}_{4}$, the most important IQA contributions to $\Delta E$ in the investigated excited states of $\mathrm{C}_{2} \mathrm{H}_{4}$ are the net energies of $\mathrm{C}$ and $\mathrm{H}$ as can be seen in the bottom of Table 4 .

\subsection{Methane}

We consider the methane molecule as another example to illustrate the IQA/EOM-CCSD method. Table 5 reports the averages for (i) the changes of QTAIM charges and delocalisation indexes as a result of different photoabsorption processes for $\mathrm{CH}_{4}$ and (ii) the corresponding IQA excitation partition energies. Before examining the results of Table 5, we point out that because $\mathcal{T}_{d}$ is not an Abelian point group, it has two- $(\mathrm{E})$ and three-dimensional $\left(\mathrm{T}_{1}\right.$ and $\mathrm{T}_{2}$ ) irreducible representations, for which the resulting reduced density functions are not completely symmetric. In other words, when the vector state is a base for any of these irreducible representations, it may occur that the calculated properties for the four hydrogen atoms are not equivalent despite the symmetry operations relating them. Hence, we have decided to present averages and sums mainly of the $\mathrm{C}-\mathrm{H}$ and $\mathrm{H} \cdots \mathrm{H}$ interactions within the methane molecule. The sum of the IQA components recovers anew the EOM-CCSD excitation 
Table 5: Average of the changes of delocalisation indexes and atomic charges (in atomic units) as a result of the photoexcitations which lead to the examined excited states of $\mathrm{CH}_{4}$. We also present the contributions to the changes of the net, classical and exchange correlation energies $(\mathrm{eV})$ as well as the comparison of the total sum of the IQA components to the excitation energy with those computed directly with EOM-CCSD theory. ${ }^{\ddagger}$

\begin{tabular}{|c|c|c|c|c|c|c|c|c|c|}
\hline State & & $1^{1} \mathrm{~T}_{2}$ & & $1^{1} \mathrm{~A}_{2}$ & \multicolumn{2}{|c|}{$1^{1} \mathrm{E}$} & \multicolumn{3}{|c|}{$2^{1} \mathrm{~T}_{2}$} \\
\hline$\langle\Delta \mathrm{DI}(\mathrm{C}, \mathrm{H})\rangle$ & -0.038 & -0.037 & -0.037 & -0.026 & -0.010 & -0.007 & -0.005 & -0.027 & -0.027 \\
\hline$\langle\Delta \mathrm{DI}(\mathrm{H}, \mathrm{H})\rangle$ & 0.048 & 0.046 & 0.046 & 0.067 & 0.036 & 0.035 & -0.014 & -0.017 & -0.017 \\
\hline$\Delta q^{\mathrm{C}}$ & -0.337 & -0.335 & -0.335 & -0.332 & -0.416 & -0.430 & -0.899 & -0.970 & -0.97 \\
\hline$\left\langle\Delta q^{\mathrm{H}}\right\rangle$ & 0.084 & 0.083 & 0.083 & 0.082 & 0.104 & 0.107 & 0.224 & 0.241 & 0.2 \\
\hline
\end{tabular}

IQA energy partition

\begin{tabular}{crrrrrrrrr}
\hline$\sum \Delta E_{\text {class }}^{\mathrm{C}, \mathrm{H}}$ & -2.47 & -2.32 & -2.32 & -2.02 & -2.18 & -2.12 & -5.12 & -5.46 & -5.46 \\
$\sum \Delta E_{\text {xc }}^{\mathrm{C}, \mathrm{H}}$ & 3.32 & 3.23 & 3.23 & 3.26 & 2.98 & 3.01 & 3.04 & 3.13 & 3.13 \\
$\sum \Delta E_{\text {class }}^{\mathrm{H}, \mathrm{H}}$ & 2.72 & 2.66 & 2.66 & 2.91 & 2.65 & 2.63 & 4.18 & 4.31 & 4.31 \\
$\sum \Delta E_{\mathrm{xc}}^{\mathrm{H}, \mathrm{H}}$ & -0.32 & -0.25 & -0.25 & -0.47 & -0.15 & -0.16 & 0.30 & 0.33 & 0.33 \\
$\Delta E_{\text {net }}^{\mathrm{C}}$ & 1.18 & 1.20 & 1.20 & 1.26 & 2.22 & 2.19 & 3.54 & 3.81 & 3.81 \\
$\sum \Delta E_{\text {net }}^{\mathrm{H}}$ & 6.09 & 6.01 & 6.01 & 6.96 & 7.24 & 7.24 & 7.34 & 7.16 & 7.16 \\
$\Delta E$ & 10.53 & 10.52 & 10.52 & 11.91 & 12.77 & 12.78 & 13.28 & 13.29 & 13.29 \\
\hline$\Delta E_{\text {MrCC }}$ & 10.53 & 10.53 & 10.53 & 11.90 & 12.77 & 12.77 & 13.27 & 13.27 & 13.27
\end{tabular}

$\ddagger$ The above differences are computed with respect to the corresponding values for the $\mathrm{CH}_{4}$ ground state: $\mathrm{DI}(\mathrm{C}, \mathrm{H})=0.798$, $\mathrm{DI}(\mathrm{H}, \mathrm{H})=0.054, q^{\mathrm{C}}=-0.083, q^{\mathrm{H}}=0.021, E_{\text {class }}^{\mathrm{C}, \mathrm{H}}=0.037, E_{\mathrm{xc}}^{\mathrm{C}, \mathrm{H}}=-0.256, E_{\text {class }}^{\mathrm{H}, \mathrm{H}}=0.003, E_{\mathrm{xc}}^{\mathrm{H}, \mathrm{H}}=-0.007$.

energies with errors no larger than $0.02 \mathrm{eV}$. Likewise, the magnitudes of the total charge in the $\mathrm{CH}_{4}$ molecule computed in different electronic states are within the interval $-0.0005 e$ to $0.001 e$.

We note that photoexcitation rises notably the electron population within the carbon atom, specially for the $2^{1} \mathrm{~T}_{2}$ degenerate states. This increase in the polarity of the $\mathrm{C}-\mathrm{H}$ bond is accompanied by an ionic stabilisation for the same interaction as reflected in the associated values of $\Delta E_{\text {class }}^{\mathrm{C}, \mathrm{H}}$. We observe the opposite behaviour for the exchange-correlation contribution for the $\mathrm{C}-\mathrm{H}$ covalent bond, $\Delta E_{\mathrm{xc}}^{\mathrm{C}, \mathrm{H}}$, in consistency with the corresponding positive values of $\langle\Delta \mathrm{DI}(\mathrm{C}, \mathrm{H})\rangle$. The inverse correlation between the atomic net charges of a two-centres, twoelectron bond and their mutual DI is easy to prove within the electron distribution function framework. ${ }^{[55]}$ As the electrons localise in one of the two centres, their atomic charges increase 
and the covariance of their populations, i.e., the DI, decrease accordingly. A different situation occurs for the $\mathrm{H} \cdots \mathrm{H}$ interactions. All the hydrogen atoms increase their positive charge as a result of photoexcitation. The QTAIM charge for a hydrogen atom in the GS of $\mathrm{CH}_{4}$ is 0.021 a.u. as reported in the footnote of Table 5 , while all the reported values of $\left\langle\Delta q^{\mathrm{H}}\right\rangle$ are positive. Consistently, there is an increase in the classical repulsion associated to the interactions among these atoms, viz., $\sum E_{\text {class }}^{\mathrm{H}, \mathrm{H}}>0$ for all the examined excited states. On the other hand, and similarly to the excited states of $\mathrm{H}_{2} \mathrm{O}$ and $\mathrm{C}_{2} \mathrm{H}_{4}$ discussed previously, photoexcitation increases the covalent character of the $\mathrm{H} \cdots \mathrm{H}$ interactions as noted in the values of $\langle\Delta \mathrm{DI}(\mathrm{H}, \mathrm{H})\rangle$ and $\sum \Delta E_{\mathrm{xc}}^{\mathrm{H}, \mathrm{H}}$. Finally, we point out that the increase of the net energy of the hydrogen atoms is the largest contribution to the excitation energy of the methane molecule. The sum of the changes of the net energy of hydrogen atoms is larger than that for the carbon atom. Thus, excited states in $\mathrm{CH}_{4}$ are alike to those of $\mathrm{N}_{2}$ in which the intra-atomic reorganisations, specially those occurring in the hydrogen atoms, govern the excitation energetics.

\subsection{Helium excimer}

As a final illustration of the application of the IQA/EOM-CCSD method, we consider the helium dimer in its ground and two excited states $1^{1} \Delta_{u}$ and $2^{1} \Sigma_{g}^{+}$. We proceeded in this way, because a photoexcitation process not only might affect considerably electronic intramolecular structures, but also could lead to important changes in intermolecular interactions. ${ }^{[56]}$ Hereof, noble gases dimers comprise examples in which electron excitations may have a strong effect on intermolecular contacts as reflected for example in the formation of excimers. In general, excimers are relevant because of their occurrence in the study of excited states in condensed media. ${ }^{56]}$ We address in this section how photoabsorption enhances the magnitude of the interaction in the helium dimer, thereby enabling the formation of the He excimer. For this purpose, we applied the IQA/EOM-CCSD methodology to several points of the potential energy curves of the above mentioned states of $\mathrm{He}_{2}$ shown in Figure 4. The minimum energy internuclear distances for the GS, $2^{1} \Sigma_{g}^{+}$and $1^{1} \Delta_{u}$ states of $\mathrm{He}_{2}$ are $2.970 \AA, 1.072 \AA$ and $1.047 \AA$ respectively. As it is well known, the minimum on the potential energy curve for $\mathrm{He}_{2}$ in its GS is extremely shallow $\left(\sim 10^{-3} \mathrm{eV}\right)$. On the other hand, the minima corresponding to the $2^{1} \Sigma_{g}^{+}$and $1{ }^{1} \Delta_{u}$ electronic states are considerably deeper $(1.03 \mathrm{eV}$ and $2.34 \mathrm{eV})$ respectively. 
Table 6: IQA partition of the excitation energy $(\mathrm{eV})$ of $1^{1} \Delta_{u}$ representation, in net as well as classical and exchange-correlation inter atomic components, as a function of distance $(\AA)$ between the helium atoms in the excimer formation. We also present the changes in the delocalisation index (au) for each point. ${ }^{\dagger}$

\begin{tabular}{lcccccc}
\hline Distance & $\Delta \mathrm{DI}(\mathrm{He}, \mathrm{He})$ & $\Delta E_{\text {class }}^{\mathrm{He}, \mathrm{He}}$ & $\Delta E_{\mathrm{xc}}^{\mathrm{He}, \mathrm{He}}$ & $2 \Delta E_{\mathrm{net}}$ & $\Delta E$ & $\Delta E_{\mathrm{MrCC}}$ \\
\hline 0.800 & 0.688 & 8.40 & -9.18 & 11.19 & 10.41 & 10.42 \\
1.047 & 0.751 & 1.68 & -3.09 & 16.10 & 14.69 & 14.70 \\
1.200 & 0.752 & 1.33 & -2.87 & 18.01 & 16.47 & 16.48 \\
1.400 & 0.719 & 1.01 & -2.55 & 19.59 & 18.05 & 18.06 \\
1.600 & 0.650 & 0.79 & -2.20 & 20.46 & 19.05 & 19.06 \\
1.800 & 0.550 & 0.63 & -1.82 & 20.86 & 19.67 & 19.68 \\
2.000 & 0.436 & 0.51 & -1.45 & 20.99 & 20.05 & 20.06 \\
2.970 & 0.188 & 0.19 & -0.61 & 20.89 & 20.47 & 20.48 \\
3.000 & 0.187 & 0.18 & -0.60 & 20.89 & 20.47 & 20.48 \\
4.000 & 0.174 & 0.05 & -0.41 & 20.81 & 20.45 & 20.46 \\
5.000 & 0.127 & 0.01 & -0.24 & 20.67 & 20.43 & 20.44 \\
\hline
\end{tabular}

$\dagger$ The corresponding values (in atomic units) for the $\mathrm{He}_{2}$ ground state are reported in the EsI of this paper.

The latter dissociation energy is comparable with that of strong intermolecular interactions such as typical H-bonds involving charged species ${ }^{[57]}$ while the second is in the order or magnitude of moderately strong covalent bonds ${ }^{[58]}$.

By virtue of the symmetry equivalence of the He atoms, the electrostatic interaction between them is always repulsive. One could conjecture that the formation of the helium excimer is related with a substantial reduction in the classical repulsion between the constituent monomers. This is however not the case as we can see in the columns corresponding to $\Delta E_{\text {class }}^{\mathrm{He} H e}$ in Tables (6) and (7). Therefore, the exchange-correlation contribution should drive the formation of the He excimer. The values of $\Delta E_{\mathrm{xc}}^{\mathrm{He}, \mathrm{He}}$ are negative for both states $1^{1} \Delta_{u}$ and $2^{1} \Sigma_{g}^{+}$in all the examined geometries of the He dimer. In other words, photoexcitation enlarges the exchange-correlation stabilisation between the helium atoms, an observation which is consistent with the fact that the DIs rise also as a result of the photoabsorption of $\mathrm{He}_{2}$. As the internuclear distance in the helium dimer is reduced, we note that both (i) the 
Table 7: IQA partition of the excitation energy $(\mathrm{eV})$ of $2{ }^{1} \Sigma_{g}^{+}$representation, in net as well as classical and exchange-correlation inter atomic components, as a function of distance $(\AA)$ between the helium atoms in the excimer formation. We also present the changes in the delocalisation index (au) for each point. ${ }^{\dagger}$

\begin{tabular}{lcccccc}
\hline Distance & $\Delta \mathrm{DI}(\mathrm{He}, \mathrm{He})$ & $\Delta E_{\mathrm{class}}^{\mathrm{He}, \mathrm{He}}$ & $\Delta E_{\mathrm{xc}}^{\mathrm{He}, \mathrm{He}}$ & $2 \Delta E_{\mathrm{net}}$ & $\Delta E$ & $\Delta E_{\mathrm{MrCC}}$ \\
\hline 0.800 & 0.552 & 27.96 & -27.47 & 11.78 & 12.27 & 12.14 \\
1.072 & 0.733 & 1.71 & -2.42 & 17.05 & 16.34 & 16.35 \\
1.200 & 0.718 & 1.57 & -2.47 & 18.54 & 17.65 & 17.66 \\
1.400 & 0.629 & 1.19 & -2.11 & 19.99 & 19.06 & 19.08 \\
1.600 & 0.465 & 0.91 & -1.61 & 20.60 & 19.91 & 19.92 \\
1.800 & 0.244 & 0.72 & -1.04 & 20.68 & 20.36 & 20.37 \\
2.000 & 0.094 & 0.58 & -0.66 & 20.63 & 20.54 & 20.55 \\
2.970 & 0.149 & 0.20 & -0.53 & 20.85 & 20.52 & 20.53 \\
3.000 & 0.151 & 0.19 & -0.52 & 20.85 & 20.52 & 20.53 \\
4.000 & 0.173 & 0.05 & -0.41 & 20.81 & 20.45 & 20.47 \\
5.000 & 0.127 & 0.01 & -0.25 & 20.67 & 20.43 & 20.44 \\
\hline
\end{tabular}

$\uparrow$ The corresponding values (in atomic units) for the $\mathrm{He}_{2}$ ground state are reported in the EsI of this paper.

classical repulsion and (ii) the exchange-correlation stabilisation between the helium atoms increase in both states associated to the helium excimer. Therefore, it is a balance between the repulsive $E_{\text {class }}^{\mathrm{He}, \mathrm{He}}$ and the attractive $E_{\mathrm{xc}}^{\mathrm{He}, \mathrm{He}}$ which leads to the minimum energy geometry. We stress the way in which electron excitations may develop a covalent contribution within an intermolecular interaction as reflected by the trends of $\Delta \mathrm{DI}(\mathrm{He}, \mathrm{He})$ and $\Delta E_{\mathrm{xc}}^{\mathrm{He}, \mathrm{He}}$ in Tables (6) and (7), an observation which is suggestive of photochemical reactions which lead to the formation of chemical bonds such as photodimerisations. Finally, we point out that the energy of the absorbed photon at the dissociation limit changes almost only the intra-atomic terms, in consistency with the notion that under these circumstances the system is comprised by an helium atom in the GS and another in the first excited state without any kind of interaction between them. 


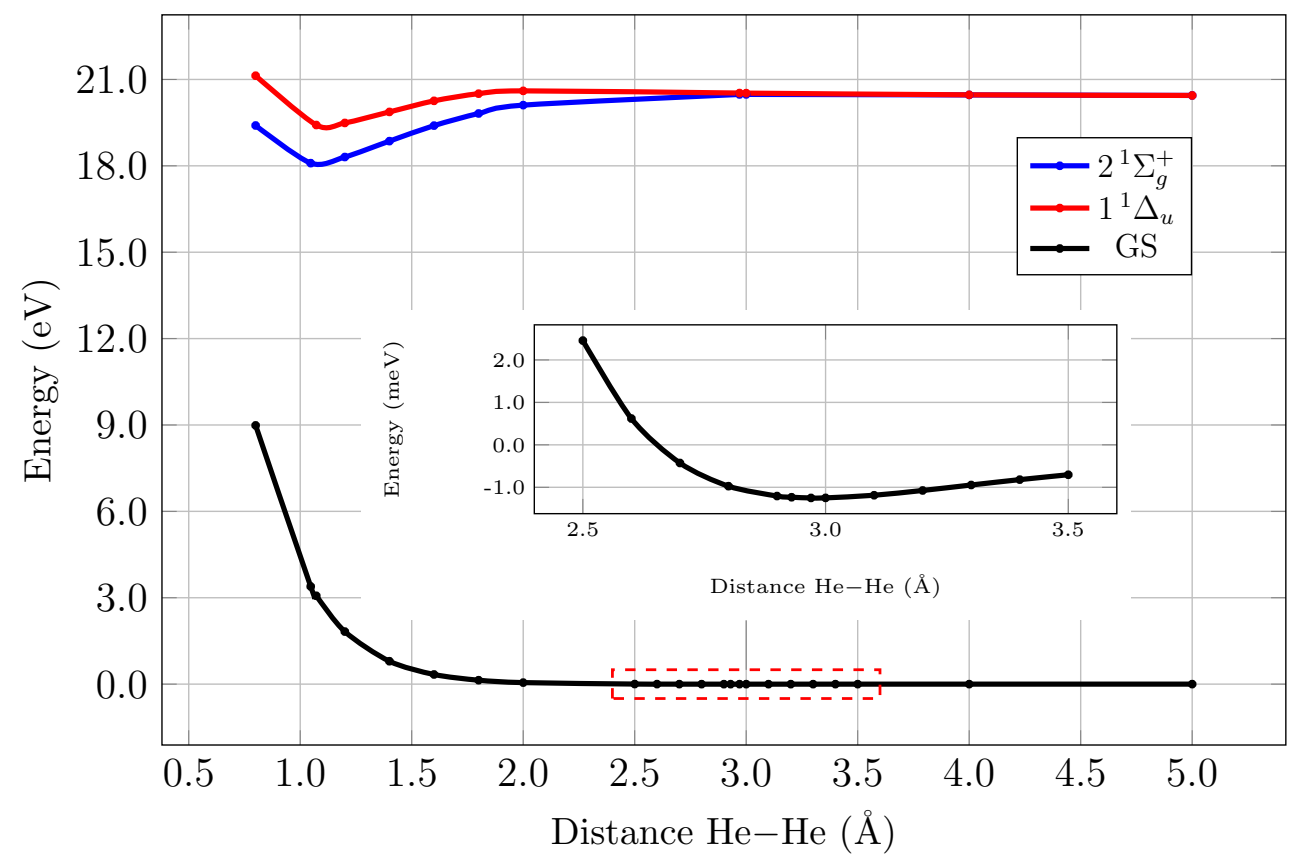

Figure 4: Potential energy curve for the He excimer formation. The electronic energy calculation for the $2{ }^{1} \Sigma_{g}^{+}$and $1{ }^{1} \Delta_{u}$ excited sates were performed withe EOM-CCSD approximation and CCSD was used for the GS energy.

\section{Concluding remarks}

We presented herein the division of EOM-CCSD excitation energies within the formalism of the IQA partition, i.e., the IQA/EOM-CCSD method. The computed one- and twoelectron density matrix elements allowed to recover the excitation energies for the addressed excited states with errors in the order of centi-electron volts. We illustrated the IQA/EOMCCSD approach with (i) diatomic, (ii) lone-pair containing and (iii) saturated and unsaturated organic molecules. This novel approach gives detailed insights of how photoexcitation affects the magnitude and the character of chemical bonds within a molecule, particularly in terms of their covalent and ionic nature. One of the most notable examples examined herein are the effects of photoexcitation on the inter-atomic classical and exchange-correlation components in the $1^{1} \Pi_{u}$ and $2{ }^{1} \Pi_{u}$ states of $N_{2}$. The IQA/EOM-CCSD also reveals a substantial increase due to photoabsorption in the covalent bonding between contiguous hydrogen atoms $\mathrm{H} \cdots \mathrm{H}$ within $\mathrm{H}_{2} \mathrm{O}, \mathrm{C}_{2} \mathrm{H}_{4}$ and $\mathrm{CH}_{4}$ as well as the helium monomers in $\mathrm{He}_{2}$, particularly in the $1^{1} \Delta_{u}$ state associated with the formation of the helium excimer. On the other hand, the comparison 
of the IQA partition between ground and excited states allowed us to get valuable insights about the most relevant components in the fundamental state of molecules, e.g. for $\mathrm{N}_{2}$ and CO. Overall, we expect that partitions of the excitation energy will be not only an important QCT approach to examine the changes in physico-chemical properties within a molecule or molecular cluster as a result of photoexcitation but also a valuable tool to get a deeper understanding of the complex phenomena which occur in the fields of photophysics and photochemistry.

\section{Acknowledgements}

We gratefully acknowledge financial support from CONACyT/Mexico (grant 253776 and Ph.D. scholarship 436154 for AFA), PAPIIT/UNAM (project IN205118) and the Spanish MINECO for grant CTQ2015-65790-P. We are also grateful to DGTIC/UNAM (grant LANCADUNAM-DGTIC-250) for computer time.

\section{References}

[1] G. Granucci, J. T. Hynes, P. Millié and T.-H. Tran-Thi, J. Am. Chem. Soc., 2000, 122, 12243-12253.

[2] Y.-F. Wang and Y.-C. Cheng, Phys. Chem. Chem. Phys., 2018, 20, 4351-4359.

[3] L. Gutierrez-Arzaluz, F. Cortes-Guzman, T. Rocha-Rinza and J. Peón, Phys. Chem. Chem. Phys., 2015, 17, 31608-31612.

[4] M. Ben-Nun and T. Martínez, Chem. Phys. Lett., 1998, 298, 57-65.

[5] J. B. Foley, S. E. Gay, M. J. Vela, B. M. Foxman, A. E. Bruce and M. R. M. Bruce, Eur. J. Inorg. Chem., 2007, 4946-4951.

[6] H.-P. Zhou, D.-M. Li, J.-Z. Zhang, Y.-M. Zhu, J.-Y. Wu, Z.-J. Hu, J.-X. Yang, G.-B. Xu, Y.-P. Tian, Y. Xie, X.-T. Tao, M.-H. Jiang, L.-M. Tao, Y.-H. Guo and C.-K. Wang, Chem. Phys., 2006, 322, 459 470.

[7] N. Abeyrathna and Y. Liao, J. Am. Chem. Soc., 2015, 137, 11282-11284.

[8] I. Ciofini, T. Le Bahers, C. Adamo, F. Odobel and D. Jacquemin, J. Phys. Chem., 2012, 116, 1194611955. 
[9] N. K. Elumalai and A. Uddin, Energy Environ. Sci., 2016, 9, 391-410.

[10] D. Kim, K. K. Sakimoto, D. Hong and P. Yang, Angew. Chem. Int. Ed., 2015, 54, 3259-3266.

[11] S. van de Linde, I. Krstić, T. Prisner, S. Doose, M. Heilemann and M. Sauer, Photochem. Photobiol. Sci., 2011, 10, 499-506.

[12] P. L. A. Popelier, in Quantum Chemical Topology: on Bonds and Potentials, Springer Berlin Heidelberg, Berlin, Heidelberg, 2005, pp. 1-56.

[13] R. F. W. Bader, Atoms in Molecules A Quantum Theory, Oxford University Press, 1995.

[14] A. Martín Pendás, M. A. Blanco and E. Francisco, J. Chem. Phys., 2006, 125, 184112-184112-20.

[15] A. Savin, R. Nesper, S. Wengert and T. F. Fässler, Angew. Chem. Int. Ed., 1997, 36, 1808-1832.

[16] E. R. Johnson, S. Keinan, P. Mori-Sánchez, J. Contreras-García, A. J. Cohen and W. Yang, J. Am. Chem. Soc., 2010, 132, 6498-6506.

[17] C. Gatti, F. Cargnoni and L. Bertini, J. Comput. Chem., 2003, 24, 422-436.

[18] E. Francisco, A. M. Pendás and M. Blanco, Comput. Phys. Commun., 2008, 178, $621-634$.

[19] M. A. Blanco, A. Martín Pendás and E. Francisco, J. Chem. Theory Comput., 2005, 1, 1096-1109.

[20] E. Francisco, A. Martín Pendás and M. A. Blanco, J. Chem. Theory Comput., 2006, 2, 90-102.

[21] J. M. Guevara-Vela, R. Chávez-Cavillo, J. Garciía-Revilla, Marco aand Hernández-Trujillo, O. Chistianse, E. Francisco, A. Martín Pendás and T. Rocha-Rinza, Chem. Eur. J., 2013, 19, 14304-14315.

[22] J. M. Guevara-Vela, E. Romero-Montalvo, V. A. Mora Gómez, R. Chávez-Calvillo, M. García-Revilla, E. Francisco, Á. Martín Pendás and T. Rocha-Rinza, Phys. Chem. Chem. Phys., 2016, 18, 19557-19566.

[23] E. Romero-Montalvo, J. M. Guevara-Vela, A. Costales, A. Martín Pendás and T. Rocha-Rinza, Phys. Chem. Chem. Phys., 2017, 19, 97-107.

[24] J. Jara-Cortés and J. Hernández-Trujillo, J. Comput. Chem., 2018, 39, 1103-1111.

[25] F. Jiménez-Grávalos, N. Díaz, E. Francisco, Á. Martín-Pendás and D. Suárez, ChemPhysChem, 2018, 19, 3425-3435.

[26] E. Romero-Montalvo, J. M. Guevara-Vela, W. E. Vallejo Narváez, A. Costales, A. M. Pendás, M. Hernández-Rodríguez and T. Rocha-Rinza, Chem. Commun., 2017, 53, 3516-3519. 
[27] D. Tiana, E. Francisco, P. Macchi, A. Sironi and Á. Martín Pendás, J. Chem. Phys., 2015, 119, 21532160.

[28] J. Jara-Cortés, J. M. Guevara-Vela, A. Martín Pendás and J. Hernández-Trujillo, J. Comput. Chem., 2017, 38, 957-970.

[29] D. Ferro-Costas, A. Pendás, L. González and R. Mosquera, Phys. Chem. Chem. Phys., 2014, 16, 92499258.

[30] T. Helgaker, P. Jørgensen and J. Olsen, Molecular Electronic-Structure Theory, John Wiley \& Sons, LTD, 2000.

[31] R. F. W. Bader, A. Streitwieser, A. Neuhaus, K. E. Laidig and P. Speers, J. Am. Chem. Soc., 1996, 118, 4959-4965.

[32] R. F. W. Bader and M. E. Stephens, J. Am. Chem. Soc., 1975, 97, 7391-7399.

[33] Á. Martín Pendás, E. Francisco, M. A. Blanco and C. Gatti, Chem. Eur. J., 2007, 13, 9362-9371.

[34] A. Szabo and N. S. Ostlund, Modern Quantum Chemistry:Introduction to Advanced Electronic Structure Theory, Dover Publications, 1996, p. 466.

[35] J. F. Stanton, J. Chem. Phys., 1993, 99, 8840-8847.

[36] F. J. Holguín-Gallego, R. Chávez-Calvillo, M. García-Revilla, E. Francisco, Á. Pendás Martín and T. Rocha-Rinza, J. Comput. Chem., 2016, 37, 1753-1765.

[37] G. E. Scuseria, C. L. Janssen and H. F. Schaefer, J. Chem. Phys., 1988, 89, 7382-7387.

[38] R. M. Parrish, L. A. Burns, D. G. Smith, A. C. Simmonett, A. E. DePrince III, U. Hohenstein, E. G.and Bozkaya, R. Sokolov, A. Yu.and Di Remigio, R. M. Richard, J. F. Gonthier, A. M. James, H. R. McAlexander, A. Kumar, M. Saitow, X. Wang, B. P. Pritchard, P. Verma, H. F. Schaefer III, K. Patkowski, R. A. King, E. F. Valeev, F. A. Evangelista, J. M. Turney, T. D. Crawford and C. D. Sherrill, Psi4 1.1: An open-source electronic structure program emphasizing automation, advanced libraries, and interoperability, 2017, www.psicode.org.

[39] A. J. Sadlej, Theor. Chem. Acc., 1992, 81, 339-354.

[40] M. Caricato, G. W. Trucks, M. J. Frisch and K. B. Wiberg, J. Chem. Theory Comput., 2010, 6, 370-383.

[41] O. Christiansen, H. Koch, P. Jørgensen and J. Olsen, Chem. Phys. Lett., 1996, 256, 185-194. 
[42] J. F. Stanton and R. J. Bartlett, J. Chem. Phys., 1993, 98, 7029-7039.

[43] J. Jara-Cortés, J. M. Guevara-Vela, Á. Martín Pendás and J. Hernández-Trujillo, J. Comput. Chem., 2017, 38, 957-970.

[44] M. Kállay, Z. Rolik, J. Csontos, P. Nagy, G. Samu, D. Mester, J. Csóka, B. Szabó, I. Ladjánszki, L. Szegedy, B. Ladóczki, K. Petrov, M. Farkas, P. D. Mezei and Hégely.B., MRCC, a quantum chemical program suite, 2013, www.mrcc.hu.

[45] M. Kállay and J. Gauss, J. Chem. Phys., 2004, 121, 9257-9269.

[46] M. A. Blanco, A. Martín Pendás and E. Francisco, J. Chem. Theory Comput., 2005, 1, 1096-1109.

[47] Á. Martín Pendás and E. Francisco, A QTAIM/IQA code (Available from the authors upon request).

[48] X. Fradera, M. A. Austen and R. F. W. Bader, J. Phys. Chem., 1999, 103, 304-314.

[49] A. Martín Pendás and E. Francisco, Phys. Chem. Chem. Phys., 2018, 20, 12368-12372.

[50] E. Francisco, A. Martín Pendás and M. A. Blanco, J. Chem. Phys., 2007, 126, 094102.

[51] M. Menéndez, R. Alvarez Boto, E. Francisco and A. Martín Pendás, J. Comput. Chem., 2015, 36, 833-843.

[52] E. I. Sánchez-Flores, R. Chávez-Calvillo, T. A. Keith, G. Cuevas, T. Rocha-Rinza and F. Cortés-Guzmán, J. Comput. Chem., 2014, 35, 820-828.

[53] M. Ben-Nun and T. J. Martínez, Chem. Phys. Lett., 1998, 298, 57 - 65.

[54] J. Moran, P. H. Cebrowski and A. M. Beauchemin, J. Org. Chem., 2008, 73, 1004-1007.

[55] A. Martín Pendás and E. Francisco, Physical Chemistry Chemical Physics, 2018, 20, 16231-16237.

[56] A. Stone, The Theory of Intermolecular Forces, Oxford university Press, 2nd edn, 2013.

[57] T. Steiner, Angew. Chem. Int. Ed., 2002, 41, 48-76.

[58] R. L. K. James E. Huheey, Ellen A. Keiter, Inorganic Chemistry: Principles of Structure and Reactivity (4th Edition), Prentice Hall, 4th edn, 1993. 